\title{
B2.5-Eunomia simulations of Magnum-PSI detachment experiments: I. Quantitative comparisons with experimental measurements
}

Citation for published version (APA):

Chandra, R., de Blank, H. J., Diomede, P., van Eck, H. J. N., van der Meiden, H. J., Morgan, T. W., Vernimmen, J. W. M., \& Westerhof, E. (2021). B2.5-Eunomia simulations of Magnum-PSI detachment experiments: I. Quantitative comparisons with experimental measurements. Plasma Physics and Controlled Fusion, 63(9), [095006]. https://doi.org/10.1088/1361-6587/ac11b6

Document status and date:

Published: 01/09/2021

DOI:

10.1088/1361-6587/ac11b6

Document Version:

Publisher's PDF, also known as Version of record

Document license:

Taverne

Please check the document version of this publication:

- A submitted manuscript is the version of the article upon submission and before peer-review. There can be important differences between the submitted version and the official published version of record.

People interested in the research are advised to contact the author for the final version of the publication, or visit the DOI to the publisher's website.

- The final author version and the galley proof are versions of the publication after peer review.

- The final published version features the final layout of the paper including the volume, issue and page numbers.

Link to publication

\footnotetext{
General rights rights.

- You may freely distribute the URL identifying the publication in the public portal. please follow below link for the End User Agreement:

www.umlib.nl/taverne-license

Take down policy

If you believe that this document breaches copyright please contact us at:

repository@maastrichtuniversity.nl

providing details and we will investigate your claim.
}

Copyright and moral rights for the publications made accessible in the public portal are retained by the authors and/or other copyright owners and it is a condition of accessing publications that users recognise and abide by the legal requirements associated with these

- Users may download and print one copy of any publication from the public portal for the purpose of private study or research.

- You may not further distribute the material or use it for any profit-making activity or commercial gain

If the publication is distributed under the terms of Article $25 \mathrm{fa}$ of the Dutch Copyright Act, indicated by the "Taverne" license above, 


\title{
B2.5-Eunomia simulations of Magnum-PSI detachment experiments: I. Quantitative comparisons with experimental measurements
}

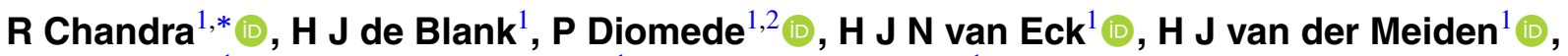 \\ T W Morgan ${ }^{1}$ (D), J W M Vernimmen ${ }^{1}$ (i) and E Westerhof ${ }^{1}$ (D) \\ ${ }^{1}$ DIFFER—Dutch Institute for Fundamental Energy Research, De Zaale 20, 5612 AJ Eindhoven, \\ The Netherlands \\ ${ }^{2}$ Faculty of Science and Engineering, Maastricht University, Zwingelput 4, ZW4, $6211 \mathrm{KH}$ Maastricht, \\ The Netherlands \\ E-mail: r.chandra@differ.nl
}

Received 17 February 2021, revised 1 June 2021

Accepted for publication 6 July 2021

Published 30 July 2021

\begin{abstract}
Detachment experiments have been carried out in the linear plasma device Magnum-PSI by increasing the gas pressure near the target. In order to have a proper detailed analysis of the mechanism behind momentum and power loss in detachment, a quantitative match is pursued between B2.5-Eunomia solutions and experimental data. B2.5 is a multi fluid plasma code and Eunomia is a Monte Carlo solver for neutral particles, and they are coupled together to provide steady-state solution of the plasma and neutral distribution in space. B2.5-Eunomia input parameters are adjusted to produce a close replication of the plasma beam measured in the experiments without any gas puffing in the target chamber. Using this replication as an initial condition, the neutral pressure near the plasma beam target is exclusively increased during simulation, matching the pressures measured in the experiments. Reasonable agreement is found between the electron temperature of the simulation results with experimental measurements using laser Thomson scattering near the target. The simulations also reveal the effect of increased gas pressure on the plasma current, effectively reducing the current penetration from the plasma source. B2.5-Eunomia is capable of reproducing detachment characteristics, namely the loss of plasma pressure along the magnetic field and the reduction of particle and heat flux to the target. The simulation results for plasma and neutrals will allow future studies of the exact contribution of individual plasma-neutral collisions to momentum and energy loss in detachment in Magnum-PSI.
\end{abstract}

Keywords: linear plasma device, detachment, fluid-kinetic code

(Some figures may appear in colour only in the online journal)

\section{Introduction}

In a tokamak fusion reactor, the heat and particle flux from the plasma core inevitably diffuse outward through the scrape-off

\footnotetext{
Author to whom any correspondence should be addressed.
}

layer (SOL) and into the divertor. It is necessary to limit the fluxes that reach the divertor materials to prevent destruction during steady-state operation. When the plasma transitions from the high-recycling regime to the detachment regime, a significant drop in ion flux to the target plates has been observed [1]. The detachment regime is identified as the 
regime when the plasma recombines before reaching the target. In addition, the detachment regime is characterized by increased volumetric losses leading to a reduction of power deposited to the target. The detachment regime is also characterized by a plasma pressure drop along the magnetic field lines from upstream to target [2]. It has been shown theoretically that momentum and power loss are both necessary for detachment to occur [3]. Interactions between charged and neutral particles are mechanisms in which the plasma loses momentum and energy, since neutral particles are unconfined by magnetic fields and able to distribute energy to the surrounding wall. In order to understand the relative contribution of plasma-neutral interactions in detachment, twodimensional steady-state code such as SOLPS have been used to simulate tokamak experiments [4-9]. It was shown that ionneutral collisions are the primary mechanism for momentum loss. This shows that inclusion of accurate ion-neutral collision physics is paramount in studying detachment. To validate the ion-neutral collision physics widely used in SOL physics models in a detached plasma scenario, a crucial step is to reproduce experimentally measured plasma parameters in a quantitative manner. This specific step can be achieved using suitable experimental platforms with high controllability and repeatability. Such qualities are reflected in linear plasma devices. Several detachment studies in linear devices have been conducted in the past and reviewed by Ohno [10]. In particular, the QED device [11] utilized the gaseous divertor concept where the surrounding gas pressure near the target is increased to terminate the plasma. It was observed that the termination of plasma is caused by the enhancement of radial heat transport due to elastic ion-neutral collisions, with the neutral particles being the primary energy carrier. A similar condition was achieved with the Magnum-PSI device [12], which has plasma parameters that are foreseen in ITER's divertor region [13]. Magnum-PSI is capable of achieving $n_{\mathrm{e}}=10^{19}$ $10^{21} \mathrm{~m}^{-3}$ and $T_{\mathrm{e}} \leqslant 5 \mathrm{eV}$, plasma particle fluxes up to $10^{25} \mathrm{~m}^{-2}$ $\mathrm{s}^{-1}$ with magnetic fields up to $2.5 \mathrm{~T}$ in steady state. Detachment has been achieved in Magnum-PSI by first establishing an attached plasma beam using specific plasma source parameters. While the source parameters remain stationary, additional gas is introduced in the chamber where the plasma is in contact the with target material. With increasing gas pressure, reductions in plasma pressure and heat flux to the target material were observed [14]. In addition, a special code was created to model the linear plasma beam called B2.5Eunomia [15]. B2.5 is a multi-fluid plasma code that solves continuity equations in a two-dimensional grid [16]. Eunomia [15] is a kinetic Monte Carlo code for neutral particles that samples sources and sinks of the plasma solution from B2.5 using collisions with test-particles in three-dimensional space. The sources and sinks are averaged into $2 \mathrm{D}$ and added as source terms in B2.5. B2.5-Eunomia is optimized for simulation geometries where the plasma to gas volume ratio is small, such as in Magnum-PSI. In addition, B2.5-Eunomia is capable to simulate vibrationally excited hydrogen molecules.

The detachment experiments in Magnum-PSI will be studied using B2.5-Eunomia. The study will be divided in two parts: the validation of the simulation results with experimental measurements, and the analysis of collisional processes in the simulation. In this paper, the first part of the study is presented. The numerical modeling of plasma detachment in Magnum-PSI using B2.5-Eunomia are compared with measured data obtained by Thomson scattering (TS) for multiple gas pressure values. In order to simulate this behavior, firstly the state of plasma without any gas puffing is replicated as close as possible in the simulation by adjusting several input parameters in B2.5-Eunomia. When the plasma state is matched, all input parameters are fixed except the neutral gas pressure in the target chamber, thus exclusively increasing the frequency of plasma-neutral interactions. The resulting simulation results are then compared to experimental measurements in the same gas pressures. These results will lay the groundwork for a detailed analysis of collisional processes involved in the momentum and energy loss during detachment, which will be presented in a following paper as the second part of the study. In addition, the simulations described here provide the systematic method of modeling other fusion relevant experiments in Magnum-PSI in order to provide plasma and neutral distribution within the vessel. Furthermore, the results of B2.5-Eunomia presented here can be used to compare with other plasma edge codes, such as SOLPS, in a different geometry, to test new implementations of physics in the code.

The paper is structured as follows: first, the experimental results are reviewed in section 2. B2.5-Eunomia and the simulation domain are described in section 3 . The input parameters within the model and their determination are explained in detail in section 4. In section 5, the method of B2.5Eunomia simulation for Magnum-PSI detachment experiments is explained as well as the comparison of the results with experimental measurements. We explore the characteristic and role of electric currents in Magnum-PSI plasmas in section 6. Finally, the detachment characteristics that are reproduced with B2.5-Eunomia simulations of Magnum-PSI detachment experiments, are discussed in section 7 for the effects of increasing gas pressure on the plasma pressure, and in section 8 for their effects to the target particle and heat flux. The conclusions and outlook of this paper are presented in section 9 .

\section{Detachment experiments in Magnum-PSI}

Detachment experiments have been carried out with multiple source parameters of Magnum-PSI plasmas [17, 18]. To help visualize these experiments, a simplified schematic of Magnum-PSI is shown in figure 1 . The vacuum chamber of Magnum-PSI is divided by skimmers into three individually pumped chambers: source, beam dump, and target chamber. TS is used to obtain electron density and temperature measurements at two locations, within the source and target chamber. The TS location near the target will be a reference coordinate in the axial direction, at $Z=0$. The other TS location is at $Z=-1.25 \mathrm{~m}$. While the TS measurement windows are fixed, the plasma source and the tungsten disc target can be adjusted in position in the $Z$-direction, thus allowing variable plasma length. The experiments use source settings described 


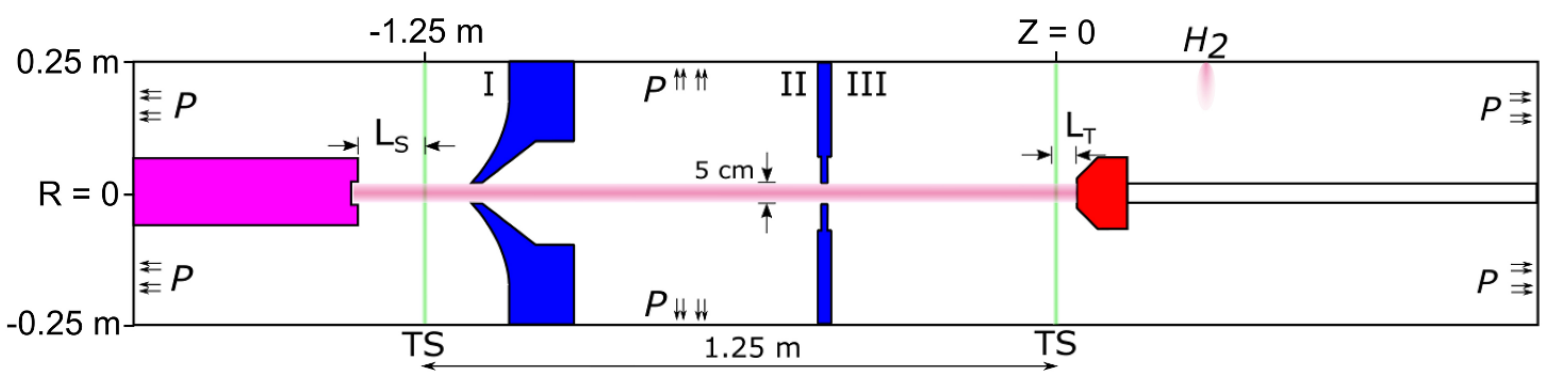

Figure 1. A simplified geometry of the Magnum-PSI [12] linear device. The device is divided into three chambers: I. source chamber, II. beam dump chamber, and III. target chamber. The chambers are separated by skimmers (blue). Each chamber is individually pumped, and the location of the pumping surfaces are denoted by $P$. There are two openings in stationary locations providing line-of-sight (green lines) for the Laser TS diagnostic. The plasma source (purple) and target holder (red) are retractable, so the distance to the TS line-of-sight, $L_{\mathrm{S}}$ and $L_{\mathrm{T}}$, can be adjusted. Additional gas can be introduced by puffing at the location denoted by $\mathrm{H}_{2}$.

Table 1. Input settings for the plasma source, $\operatorname{target}\left(L_{\mathrm{T}}\right)$ and source $\left(L_{\mathrm{S}}\right)$ locations, during Magnum-PSI detachment experiments. $F_{\mathrm{S}}$ denotes the $\mathrm{H}_{2}$ feed into the plasma source, $I_{\mathrm{S}}$ denotes the plasma source current. The pumping speed are denoted in $\%$ indicating the fraction of the pump capacity. The roman numerals denote the chambers shown in figure 1 .

\begin{tabular}{lll}
\hline Experiment settings & Low density & High density \\
\hline$F_{\mathrm{S}}(\mathrm{slm})$ & 4 & 7 \\
$I_{\mathrm{S}}(\mathrm{A})$ & 120 & 175 \\
$L_{\mathrm{T}}(\mathrm{cm})$ & 8.92 & 2.92 \\
$L_{\mathrm{S}}(\mathrm{cm})$ & 0 & 10 \\
Pump speed I, II (\%) & 100 & 82 \\
Pump speed III $(\%)$ & 100 & $82.25^{\mathrm{a}}$ \\
\hline
\end{tabular}

${ }^{a}$ Pump speed changed to attain higher neutral pressure at chamber III.

in table 1. During these experiments the magnetic field is set to $1.2 \mathrm{~T}$. While these source settings remain fixed, additional gas pressure is introduced in the target chamber, resulting in a reduction of plasma pressure. When the target chamber neutral pressure is increased, the source and beam dump chamber pressures are observed to be unchanged. The electron temperature and density are radially measured using TS at $Z=0$. The peak values of the measured profiles are shown in figure 2 . The two different source settings are chosen based on the resulting variety of plasma condition. The key differing aspects are the difference in initial electron density and its progression toward the high gas pressure shown in figure 2(a). Hence from this point forward, the case with $F_{\mathrm{S}}=7 \mathrm{slm}$ and $I_{\mathrm{S}}=175 \mathrm{~A}$ will be referred to as the high density case, and the case with $F_{\mathrm{S}}=4 \mathrm{slm}$ and $I_{\mathrm{S}}=120 \mathrm{~A}$ will be referred to as the low density case. The additional gas pressure is realized by $\mathrm{H}_{2}$ puffing of varying flow within the target chamber. One exception is during the high density case, where initially only the pumping speed at the target chamber is lowered from $82 \%$ to $25 \%$ to increase the gas pressure from 0.45 to $0.9 \mathrm{~Pa}$. Gas puffing is used on the transition from $0.9 \mathrm{~Pa}$ to higher pressures. In order to model these experiments using B2.5-Eunomia, information of $T_{\mathrm{e}}$ and $n_{\mathrm{e}}$ at the source TS location is required as boundary conditions. Unfortunately, within the same experiments these data are absent. Hence, we assume that different experiments using the same source settings listed in table 1 yielded the same plasma parameters at the source TS location. The measured $T_{\mathrm{e}}$ and $n_{\mathrm{e}}$ profiles used as boundary conditions for B2.5-Eunomia are shown in figure 3.

The detached plasma condition in Magnum-PSI is characterized by the location of the H- $\alpha$ emission. The plasma is considered 'attached' when the light emission is very localized directly near the material surface. As the gas pressure is increased, more of the plasma volume is visibly emitting light. If the pressure is high enough, the bright plasma volume can be seen to move away from the target, and is visibly 'detached' (see figure 2 of [18]). The transition from attached to detached is visibly continuous and proportional to the gas pressure. This is also reflected in the electron density and temperature shown in figure 2. In this paper, the plasma parameters that are discussed lies in the middle of this transition regime. It is important to note that the Magnum-PSI plasma discharges discussed here are already in a low temperature regime comparable to the detachment regime in tokamaks. At these temperatures the ionization rate is relatively negligible compared to the plasma flux from the cascaded arc source.

\section{B2.5-Eunomia}

The experiments are modeled using the coupled fluid-kinetic Monte Carlo code B2.5-Eunomia. B2.5 is a two-dimensional multi-fluid code that solves the continuity equation for particle, momentum and energy [16]. Eunomia is a threedimensional Monte Carlo kinetic model for neutral particles [15]. To help with understanding the coupling process of these two codes, the simulation grid is shown in figure 4. Cylindrical symmetry is assumed with the axis at $R=0$. The B2.5 simulation domain encompasses the plasma beam and extends axially from the source, which is defined by the location of TS near the source, to the target boundary. The plasma beam radius is limited to the skimmer opening width, with a radius of about $r \approx 0.025 \mathrm{~m}$. However, the B2.5 domain spans only to $r \approx 0.02 \mathrm{~m}$. This small gap is necessary to obtain a functional triangular grid from B2.5-Eunomia as shown in figure 4. Nevertheless, for the experiments discussed in this paper, most of the plasma bulk resides inside $r=0.02 \mathrm{~m}$ as shown in figure 3 . The exclusion plasma within $0.02<r<0.025 \mathrm{~m}$ is assumed to have little effect on the final plasma solution. For Eunomia, the domain includes the plasma beam and the rest of the 


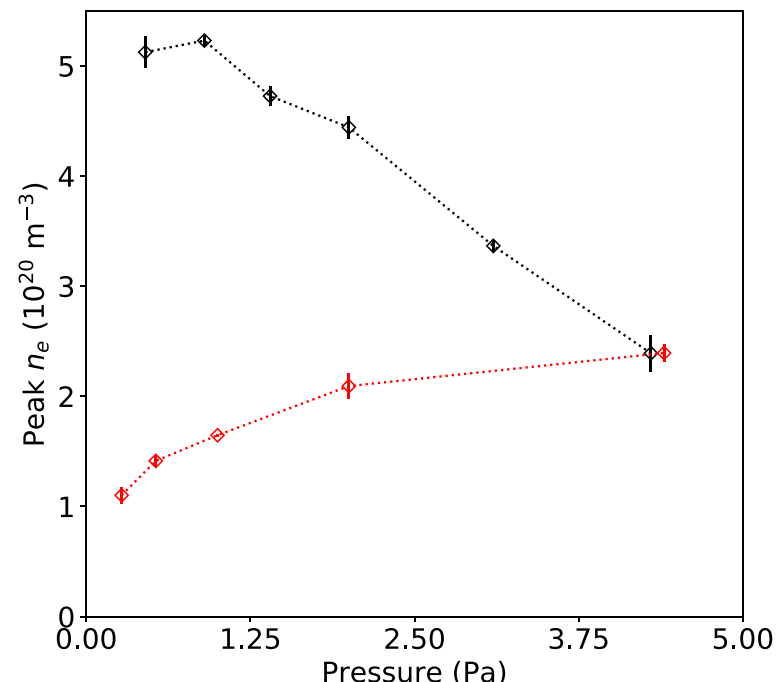

(a)

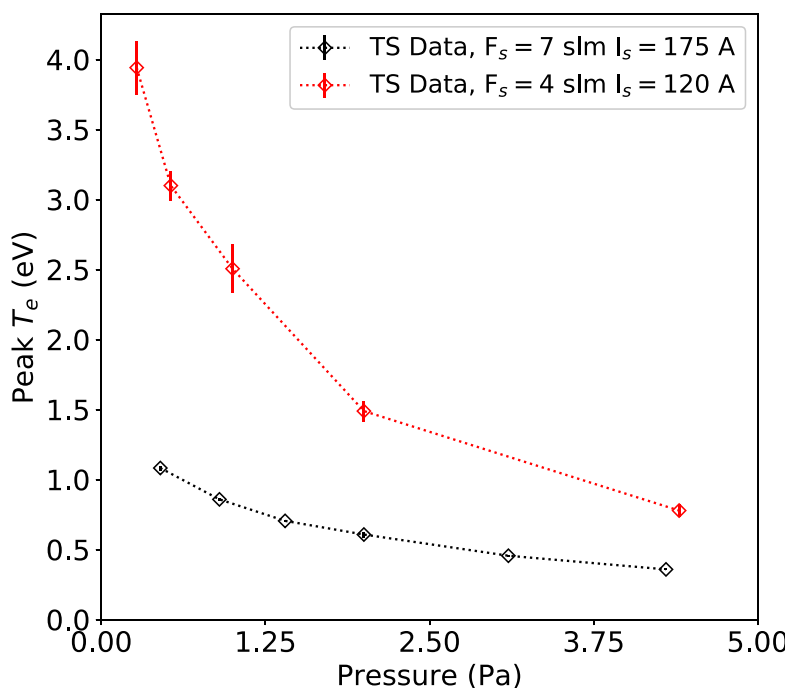

(b)

Figure 2. (a) Peak electron density and (b) temperature measured at $Z=0$ for the two different detachment experiments in Magnum-PSI. The two cases are chosen based on the different plasma regime shown here. Experiments producing black data points are referred to as the high density case [17], and red data points as the low density case [18].

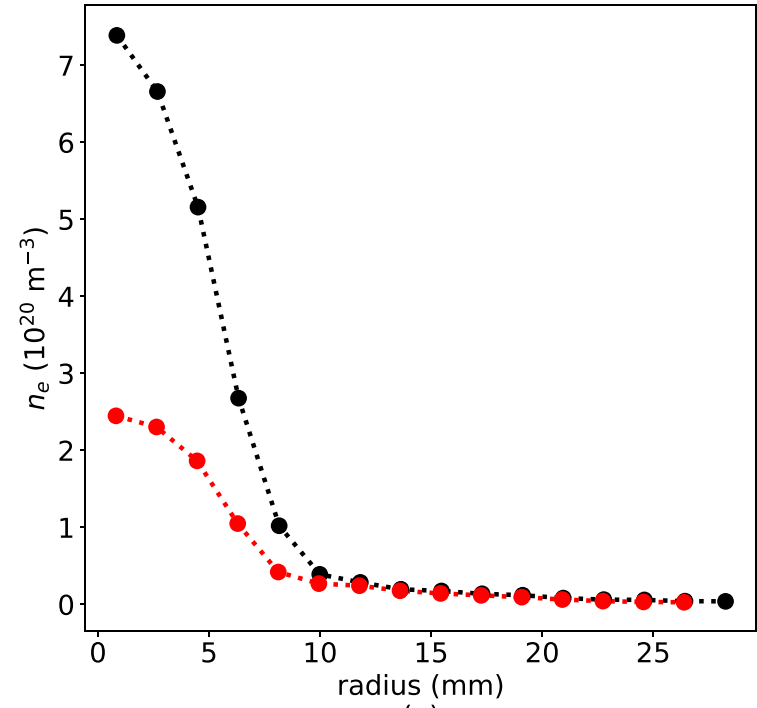

(a)

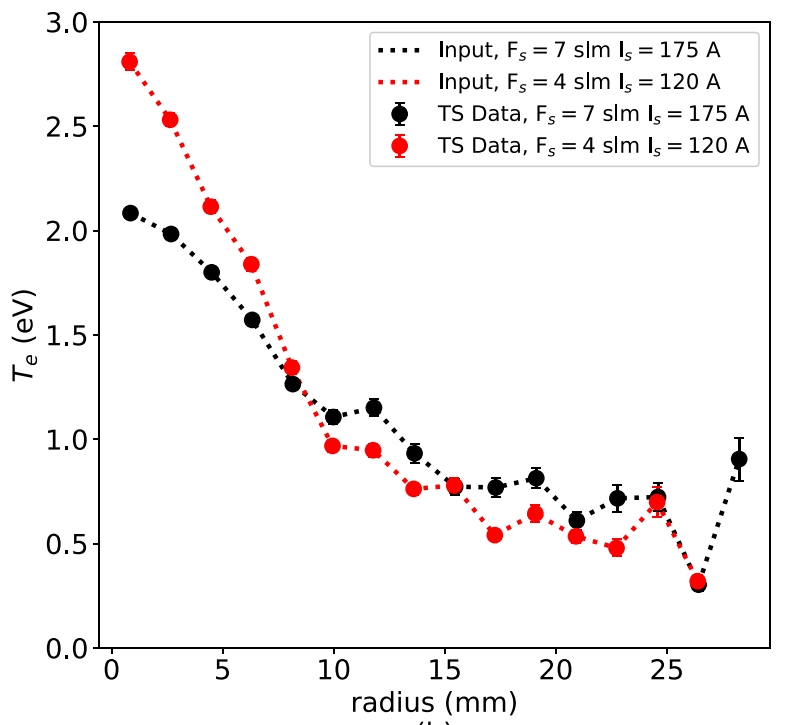

(b)

Figure 3. (a) Electron density and (b) temperature used as input parameters in B2.5-Eunomia on the source boundary. The TS data are measured values at the TS location near the source, or at $Z=-1.25 \mathrm{~m}$. The values that are used for the B2.5-Eunomia inputs are measurement data points and the linearly interpolated points in between.

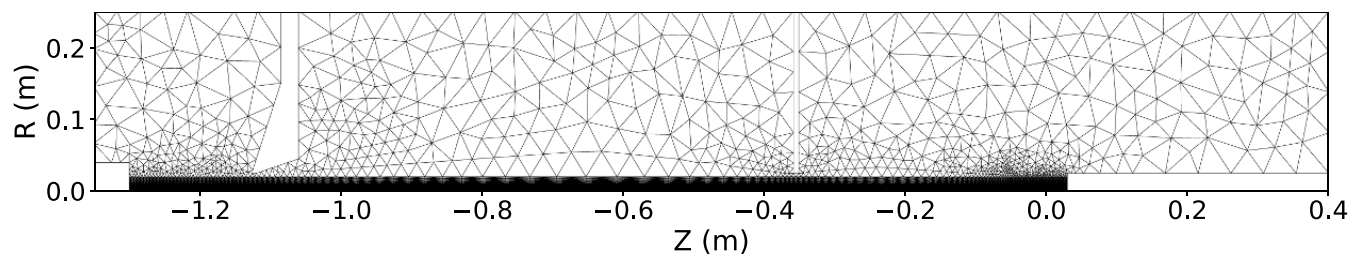

Figure 4. 2D simulation grid of Magnum-PSI. Regions with $R<0.02 \mathrm{~m}$ are dark due to the high resolution of the grid. This region corresponds to the plasma domain where B2.5 fluid code is used to solved the plasma continuity equations. Eunomia uses a 3D structure that is generated by rotating this entire grid on the $R=0$ axis. The source boundary is located near the $Z=-1.2 \mathrm{~m}$ mark, while the target boundary is located near the $Z=0$ mark. 
vessel shown in figure 4. B2.5 provides the $2 \mathrm{D}$ symmetric plasma information, namely density, flow velocity and temperature. In Eunomia, neutral test particles interact with the plasma by colliding with randomly generated plasma particles from the Maxwellian solution provided by B2.5. From these interactions, source and sink terms for the plasma are collected and provided back to B2.5. The reader is referred to [19] and the references therein for complete descriptions of B2.5, and $[15,20]$ for descriptions of Eunomia. One important note is that the simulations in this paper are conducted with B2.5 from SOLPS5.0 [21] with additional features for the boundary conditions for modeling linear devices, namely the ability to use radial profiles of density, temperature, and potential. All the collisions introduced in [15] are included in the simulations of this paper, with the addition of dissociation of $\mathrm{H}_{2}$ molecules in their vibrationally excited states $(v=0-14)$, where $v$ is the vibrational quantum number. The complete collision list implemented here is shown in appendix A.

\section{Determining input parameters for the zero puffing condition}

In order to simulate the plasma beam in Magnum-PSI, B2.5Eunomia requires specification of the plasma and neutral input parameters. The plasma and neutral inputs can further be separated into fixed and 'free' categories. Fixed inputs are experimentally measured values that can be implemented directly in the simulation, while for free inputs there are no experimental measurements data available directly.

\subsection{Fixed input parameters}

The radial electron density and temperature profile at the source boundary can be obtained using the TS system and linearly interpolated to obtain the values in between, as shown in figure 3. These profiles can be directly used as the plasma source boundary in B2.5. The neutral gas pressures are measured within individual Magnum-PSI chambers. To increase gas pressure, an additional $\mathrm{H}_{2}$ neutral source is employed in the location $R, Z=(0.25,0.3) \mathrm{m}$. Whereas the experiment maintains a certain pump speed while increasing the gas puffing flow, Eunomia adjusts absorption probabilities on pumping surfaces while keeping the same particle flux from the puffing. The absorption probabilities are determined using separate feedback loops for each of the chambers with the experimentally measured gas pressure as a control parameter, hence the same gas pressure can be achieved in the simulation. The exception is the high density case mentioned in section 2 where it is accommodated in the simulation by turning off the extra neutral source to achieve 0.45 and $0.9 \mathrm{~Pa}$. We specify the plasma-neutral interface i.e. the B2.5 boundary at $r=0.02$ with an e-folding length for density $\lambda_{\mathrm{n}}$ and temperature $\lambda_{\mathrm{T}}$. The values are derived from the radial $n_{\mathrm{e}}$ and $T_{\mathrm{e}}$ profiles at $Z=0$ from the lowest pressure data points for each source setting shown in figure 2. Lastly, the hydrogen neutral particle flux from the plasma source due to imperfect ionization can be approximated. Since the plasma source boundary starts on the TS measurement location, there is some plasma volume between the simulation domain and the source hole. Here it is assumed that the neutrals diffuse isotropically, and so the flux of hydrogen neutrals at the simulation domain is approximated by the area ratio between the disc cap and the envelope of a right cylinder. The total neutral flux entering the simulation domain is equal to the total gas flow from the source minus the plasma particle flux. However, the plasma particle flux is unknown due to the missing information regarding the plasma velocity. While the total gas flow from the source is known (see table 1 ), the degree of ionization can vary between $10 \%$ and $20 \%$ depending on the source parameters [22]. As an approximation, the ionization degree is assumed to be $10 \%$ for the calculation of total neutral flux. Fixed boundary parameters also include conditions that are chosen to model physical boundaries, for example the sheath boundary condition and floating electric potential at the target, and zero flux at the axis of cylindrical symmetry.

\subsection{Free input parameters}

Other parameters can be regarded as free and these parameters are adjusted to match the simulation results with experimental measurements of electron density and temperature, specifically the radial profiles of $n_{\mathrm{e}}$ and $T_{\mathrm{e}}$ at the TS location near the target. For example, the plasma potential at the source is required to include the electric current. This information can be obtained by measuring the plasma rotation caused by $\mathbf{E} \times \mathbf{B}$ drift. However, such measurement with sufficient accuracy is unavailable for Magnum-PSI at the time this paper is written. There are measurements from the Pilot-PSI plasma source [23], which is highly similar to the Magnum-PSI plasma source. These measurements are used to define only the characteristic shape of the potential profile, since the magnitude of the potential depends on the cathode voltage within the plasma source, and the potential difference between the cathode and the source boundary is unknown. The height of the profile is used as a parameter to control the amplitude of the electric current, which indirectly influences the electron temperature via Joule heating. The resulting profiles used for each case mentioned in table 1 are shown in figure 5. Other important parameters are the plasma transport coefficients perpendicular to the magnetic field. The transport coefficients are: particle diffusivity $D_{\mathrm{n}}$, electron heat diffusivity $\chi_{\mathrm{e}}$, and ion heat diffusivity $\chi_{\mathrm{i}}$. In B2.5 these parameters are considered anomalous and described ad-hoc, mainly for particle and heat diffusion. Simulations of Pilot-PSI using SOLEDGE2D-Eirene indicate some sensitivity of radial plasma transport to particle diffusion coefficient [24]. In order to gain some insight into choosing radial transport coefficients, first we calculate the lower limits of classical diffusion using the random walk approximation. Using the Braginskii formulation for electron collision time and ion collision time [25], we determine the lower limits of our diffusion constants using the peak temperature and density values from target TS measurements. From this limit we use incremental values of the coefficient until a good match is achieved between simulation results and experimental measurements. Other simulation parameters that can be adjusted to 


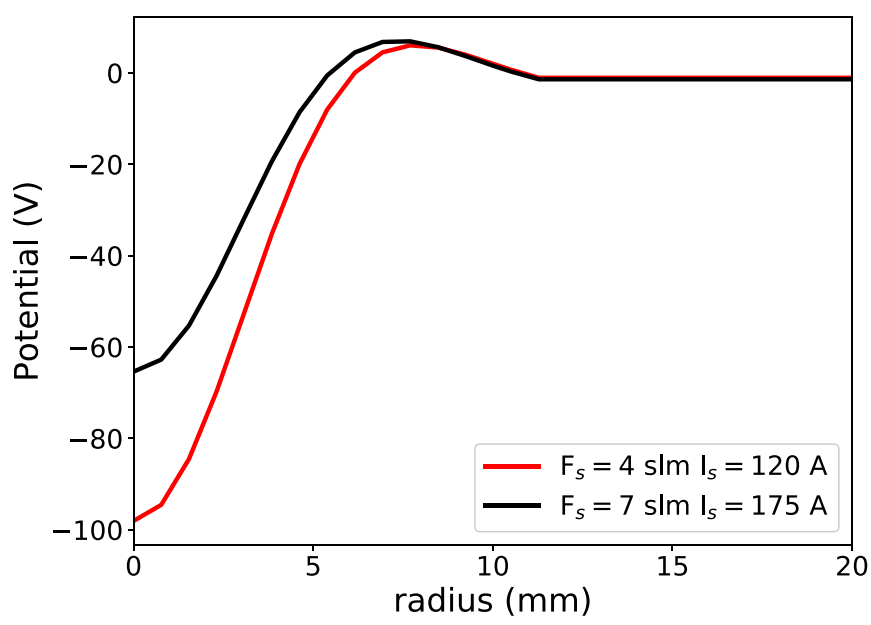

Figure 5. Potential profile used at the source boundary for the two cases.

match the experiments are the surface recombination probability of $\mathrm{H}^{+}$at the target and the recombination probability of hydrogen atoms into molecules, or $\mathrm{H}_{2}$ association, on the cold wall surfaces.

\section{B2.5-Eunomia simulations of detachment experiments in Magnum-PSI}

\subsection{Adjusting free parameters to match zero puffing condition}

The required free parameters discussed in the previous section are adjusted so that the simulation results match the measured heights and widths of the radial $n_{\mathrm{e}}$ and $T_{\mathrm{e}}$ profiles at $Z=0 \mathrm{~m}$ at the lowest neutral gas pressure i.e. when there is no gas puffing. The chosen parameter values are shown in table 2 . In addition, zero gradient boundary condition is used for the plasma velocity at the source. The resulting comparisons are shown in figure 6. For the high density case (shown in black in figure 6), we obtain reasonably good agreement for electron temperature using the classical limit for radial diffusion coefficient. However, there is around $20 \%$ difference for the electron density profile. Further adjustment of the particle diffusion coefficient could bring the peak value of $n_{\mathrm{e}}$ closer to TS measurement, however there are several effects in doing so. Firstly the width of the $n_{\mathrm{e}}$ profile will increase. As shown in figure 6 the current solution is slightly wider than the measured profile, thus increasing particle diffusion coefficient will further deviate the solution from experimental measurements. In addition, while the peak value of $n_{\mathrm{e}}$ would decrease, the peak value of $T_{\mathrm{e}}$ will increase, thus necessitating further increase of $\chi_{\mathrm{i}}$ and $\chi_{\mathrm{e}}$ and the same deviation in profile width will occur for $T_{\mathrm{e}}$. Furthermore, increasing $\mathrm{H}_{2}$ association probability on the outer vessel walls $(r=0.25 \mathrm{~m})$ does not yield significant changes to the $n_{\mathrm{e}}$ profiles. This discrepancy can be associated with the plasma influx at the source where we employ the homogeneous Neumann boundary condition for plasma velocity. The velocity solution might be higher compared to reality resulting
Table 2. Input parameter values for B2.5-Eunomia simulations presented in this paper.

\begin{tabular}{llc}
\hline Input parameters & Low density & High density \\
\hline Peak source potential $(\mathrm{V})$ & -98 & -65 \\
$\lambda_{\mathrm{n}}(\mathrm{m})$ & 0.01 & 0.0043 \\
$\lambda_{\mathrm{T}}(\mathrm{m})$ & 0.015 & 0.014 \\
$D_{\mathrm{n}}\left(\mathrm{m}^{2} \mathrm{~s}^{-1}\right)$ & 0.012 & 0.084 \\
$\chi_{\mathrm{i}}\left(\mathrm{m}^{2} \mathrm{~s}^{-1}\right)$ & 1.8 & 0.3174 \\
$\chi_{\mathrm{e}}\left(\mathrm{m}^{2} \mathrm{~s}^{-1}\right)$ & 1.8 & 0.03174 \\
Target surface recomb. & $100 \%$ & $100 \%$ \\
$\mathrm{H}_{2}$ association & $10 \%$ & $10 \%$ \\
$\mathrm{H}_{2}$ puffing $\left(\mathrm{s}^{-1}\right)$ & 10 & 1021 \\
\hline
\end{tabular}

in higher particle content. Without any information regarding the real velocity profile at the source, prior effort in imposing a Dirichlet type boundary for plasma velocity failed to produce simulations that numerically converged. As such, we conclude that for the high density case this solution has the closest equivalency to experimental $n_{\mathrm{e}}$ and $T_{\mathrm{e}}$ profiles given the possible input parameters.

For the low density case (shown in red in figure 6), a good agreement is obtained between simulation and experimental measurements for both $n_{\mathrm{e}}$ and $T_{\mathrm{e}}$ profiles. In these results, the solution was not obtained using only the classical limit of radial diffusion coefficient. To illustrate this, we simulate this case with several adjustment in radial diffusion coefficients as shown in figure 7 . We first simulate the condition using classical diffusion limit and a potential boundary value that is used for the high density case (with minimum of $-65 \mathrm{~V}$ ). The simulation using classical diffusion limit (shown in blue in figure 7) is unable to provide a matching solution, with temperature values highly peaked at the center. In order to increase the overall electron temperature, we further decrease the potential boundary minimum to $-98 \mathrm{~V}$ and adjust the radial diffusion coefficients to $D_{\mathrm{n}}=0.06$ and $\chi_{\mathrm{i}}=\chi_{\mathrm{e}}=0.6 \mathrm{~m}^{2} \mathrm{~s}^{-1}$. At this point the temperature values for $r>5 \mathrm{~mm}$ are closer to experimental data, however the central values are still peaked (shown in black in figure 7 . Here we increase the $\chi_{i}$ value and decrease $D_{\mathrm{n}}$ in order to bring the solution closer to experimental data for both $n_{\mathrm{e}}$ and $T_{\mathrm{e}}$. An adjustment of $\chi_{\mathrm{i}}=\chi_{\mathrm{e}}=1.8 \mathrm{~m}^{2} \mathrm{~s}^{-1}$ is necessary to produce the $T_{\mathrm{e}}$ profile shown in figure 6 . With these parameters we are able to produce plasma profiles similar to the ones observed in experiments, and as such this solution will be used for the low density case for comparisons at higher neutral densities.

Two very different values of the radial heat diffusivity are used for the low and high density cases to match the electron density and temperature profiles of the simulation with experiments. While they are necessary to provide the match, we lack the understanding of the nature of radial transport in Magnum-PSI needed to conclude which values are realistic. While extensive studies have been performed about crossfield transport in tokamaks, in general, a reliable systematic approach in modeling Magnum-PSI plasmas with B2.5Eunomia requires more diagnostics than were present in the experiments mentioned here. 


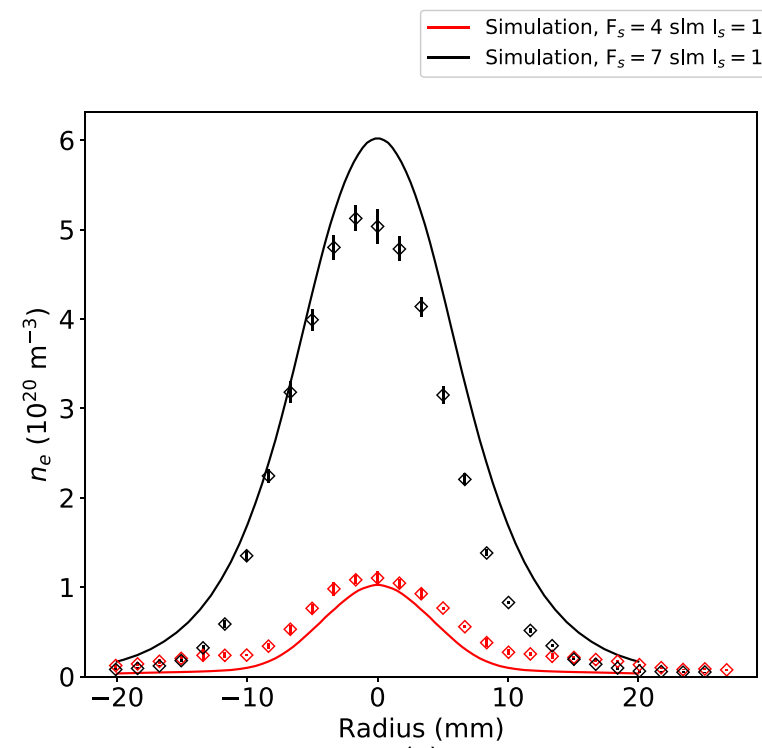

(a)
TS Data, $F_{s}=4 \mathrm{sim} \mathrm{I} s=120 \mathrm{~A}$

TS Data, $F_{s}=7 \mathrm{slm} \mathrm{I} \mathrm{I}_{s}=175 \mathrm{~A}$

Figure 6. Radial profiles of (a) electron density and (b) temperature from simulations for the high density case at $P_{\mathrm{n}}=0.45 \mathrm{~Pa}$ and low density case $P_{\mathrm{n}}=0.27 \mathrm{~Pa}$, at $Z=0$. The TS data are measured at the same location in the experiment.

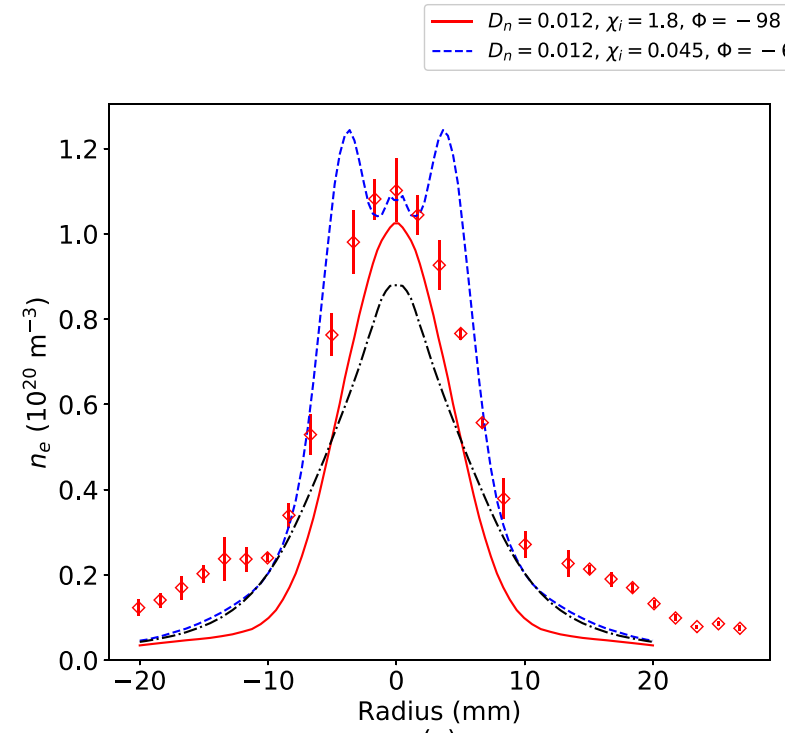

(a)

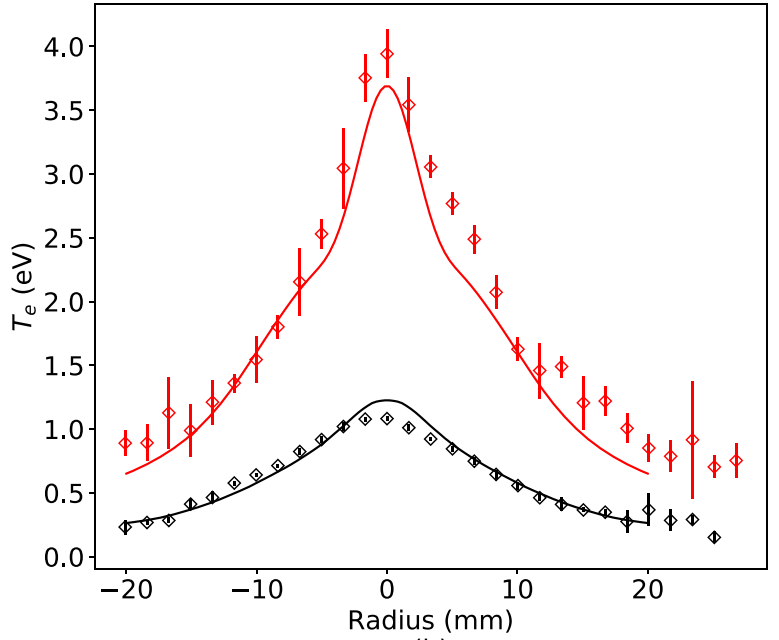

政 $0.06, \chi_{i}=0.6, \Phi=-98 \mathrm{~V}$

--- $D_{n}=0.012, \chi_{i}=0.045, \Phi=-65 \mathrm{~V} \quad \phi \quad$ TS data

Figure 7. Radial profiles of (a) electron density and (b) temperature at $Z=0$ for the low density case at $P_{\mathrm{n}}=0.27 \mathrm{~Pa}$. Different radial diffusion coefficients and potential boundary values are used to obtain the results. The blue curve is obtained when using the same classical diffusion approach as in the high density case. The black curve is obtained by increasing the diffusion coefficients and the potential boundary value as an attempt to match electron temperature values measured by TS. The best match shown by the red curve is obtained using a highly anomalous value of $\chi_{\mathrm{i}}$.

\subsection{Comparison in higher neutral gas pressures}

In the previous section we explained the method of adjusting free input parameters in the simulation in order to attain a plasma solution sufficiently close to the measured plasma state in experiments during zero gas puffing. Now, we will use the same plasma solution as our initial condition for the higher neutral pressures. All parameters except the target chamber neutral pressure will remain fixed in the simulation. The neutral pressure is adjusted according to experimental values shown in figure 2 . The plasma velocity profile at the source

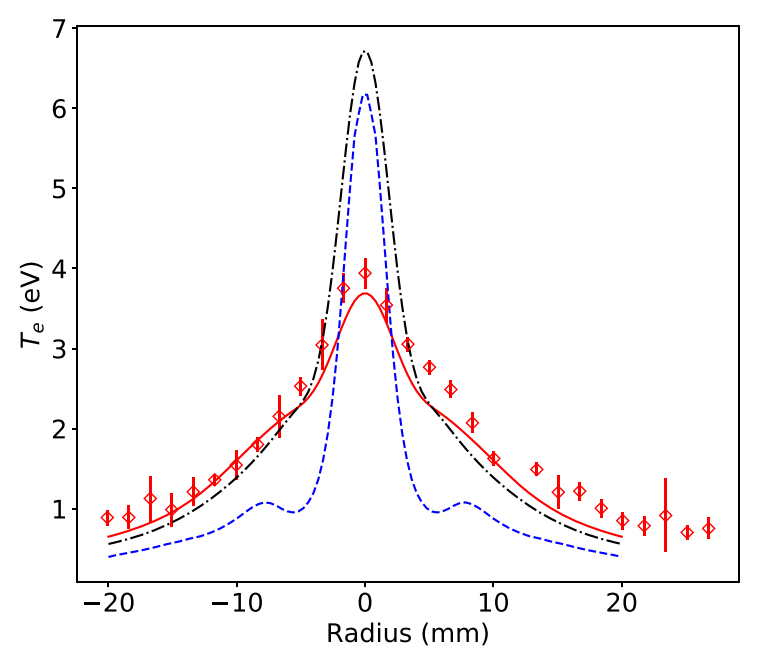

(b) 


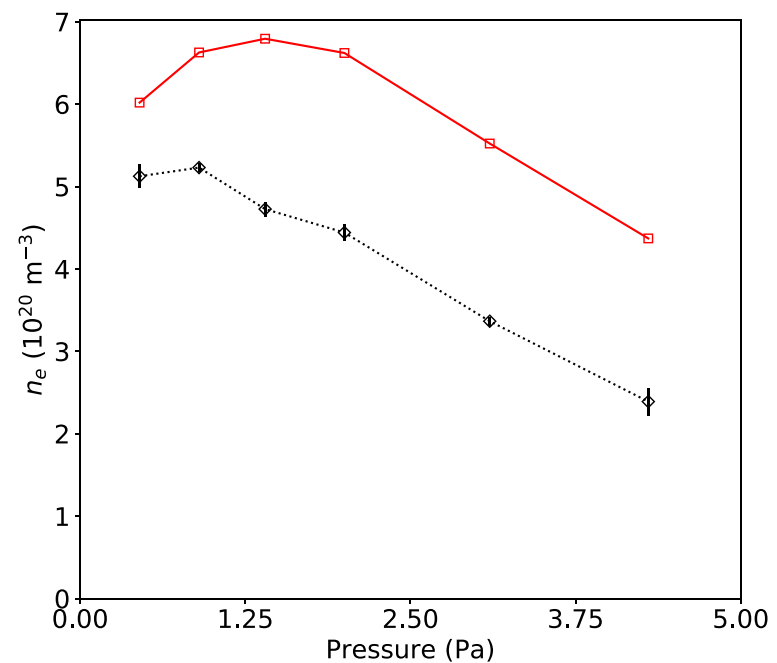

(a)

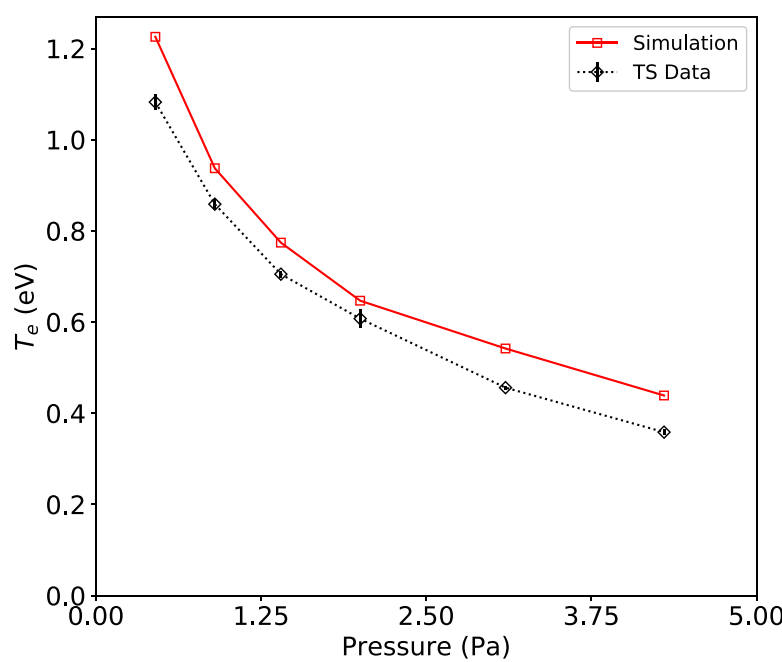

(b)

Figure 8. Comparison of peak (a) electron density and (b) temperature values at $Z=0$ between B2.5-Eunomia solutions and TS measurements for the high density case. The data points correspond to $P_{\mathrm{n}}=0.45,0.9,1.4,2.0,3.1$, and $4.3 \mathrm{~Pa}$ in the target chamber.

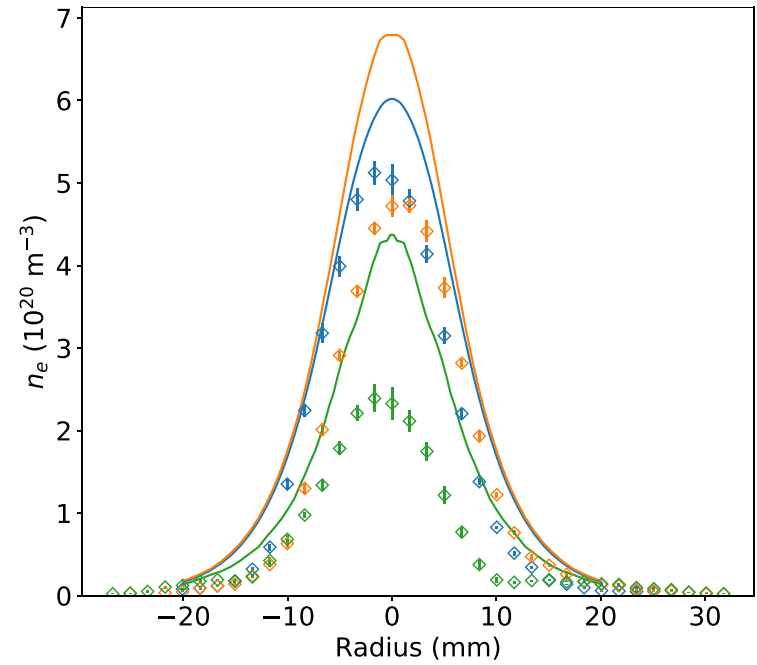

(a)

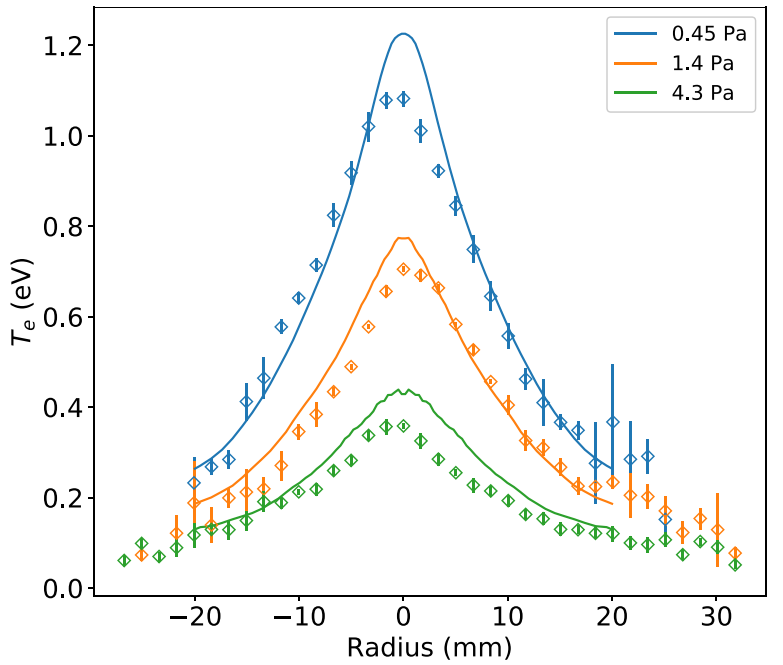

(b)

Figure 9. (a) Electron density and (b) temperature radial profiles from the high density case at $Z=0$ for $P_{\mathrm{n}}=0.45,1.4$ and $4.3 \mathrm{~Pa}$.

For the high density case, the pressure inside the target chamber is varied from 0.45 to $4.3 \mathrm{~Pa}$. The peak values of $n_{\mathrm{e}}$ and $T_{\mathrm{e}}$ profiles at the TS location near the target, or $Z=0$, are extracted for each pressure points from simulations and experimental measurements and shown in figure 8 . We observe good agreement both in trend and value of the peak electron temperatures, with the difference between simulation and experiment widening at larger gas pressures. However, there is a significant difference within the peak density comparison. Particularly, the rollover point where the density starts to decrease occurs at $1.4 \mathrm{~Pa}$, while in the experiment the rollover already occurs at $0.9 \mathrm{~Pa}$. To further elucidate the effects of pressure increase, we show the radial profile comparison for pressures $0.45,1.4$ and 4.3 $\mathrm{Pa}$ in figure 9. Again here a good agreement in trend and value of the electron temperature profiles is observed. However, significant differences in values are exhibited between simulation and experimental measurement for the electron density. The initial mismatch of electron density during zero gas puffing is observed to be exacerbated when the plasma is driven to detachment. The overall larger electron density and the delay in rollover reinforces the notion that the plasma velocity at the source boundary, and therefore the particle flux, is larger in the simulation than in the experiment. It is important to note that, while the electron density has different values as shown from figure 8, the trend in width is conserved as shown in figure 9 i.e. the plasma does not appear to be widening in experiments and in the simulations.

For the low density case, the target chamber pressure is varied from 0.27 to $4.4 \mathrm{~Pa}$. The peak values of $n_{\mathrm{e}}$ and $T_{\mathrm{e}}$ profiles at the TS location near the target, or $Z=0$, are extracted for each pressure point from simulations and experimental measurements and shown in figure 10. Here we can observe excellent agreement both in trend and value of peak $T_{\mathrm{e}}$ values, with the exception of the highest gas pressure value. The electron temperature is underestimated in the highest neutral pressure point in the simulation. A slightly different trend 


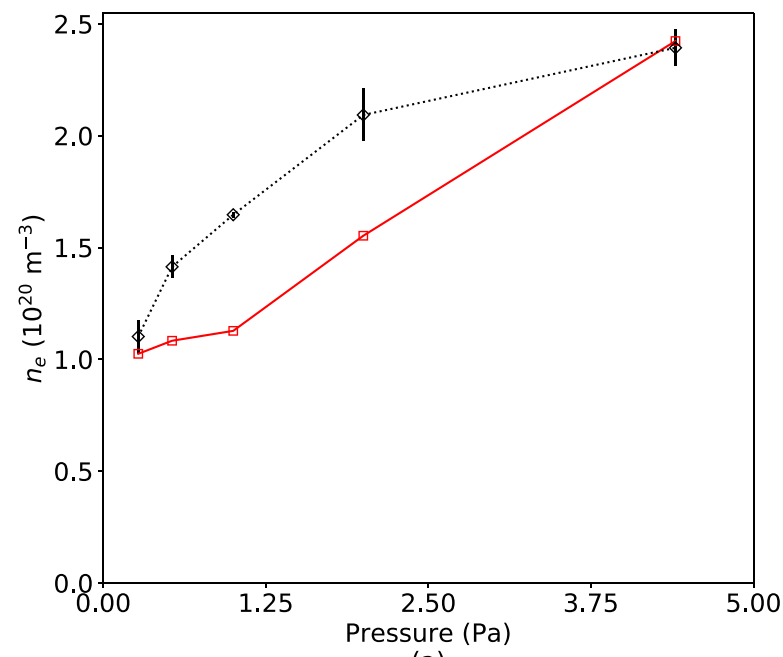

(a)

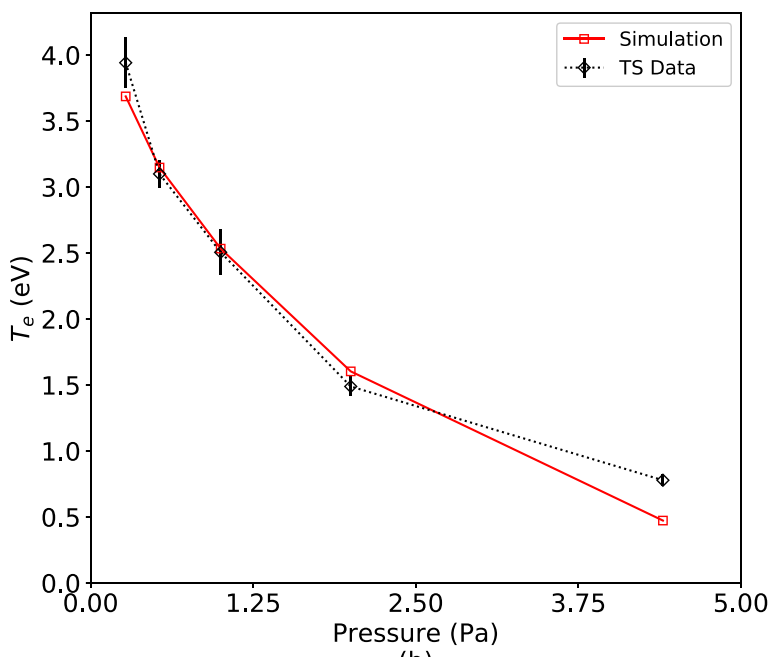

(b)

Figure 10. Comparison of peak (a) electron density and (b) temperature values at $Z=0$ between B2.5-Eunomia solutions and TS measurements for the low density case. The data points correspond to $P_{\mathrm{n}}=0.27,0.53,1.0,2.0$, and $4.4 \mathrm{~Pa}$ in the target chamber.

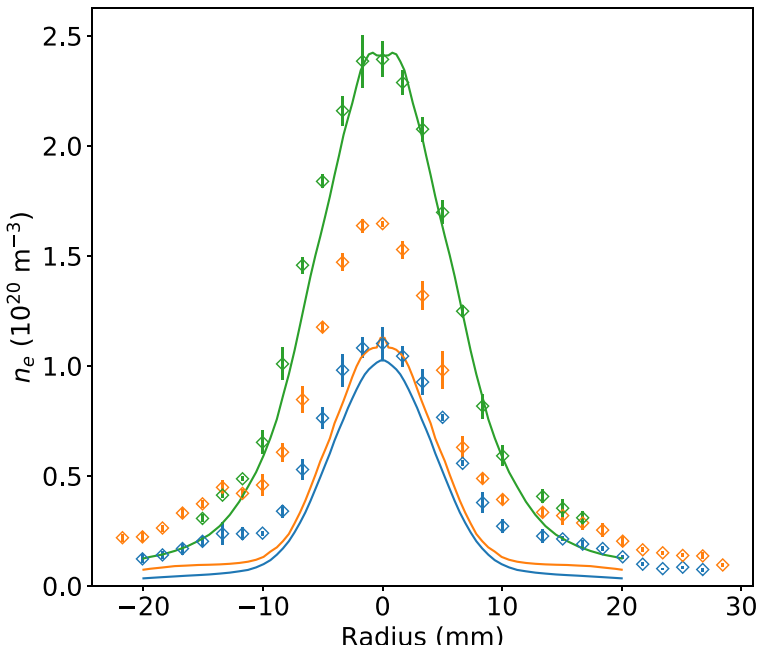

(a)

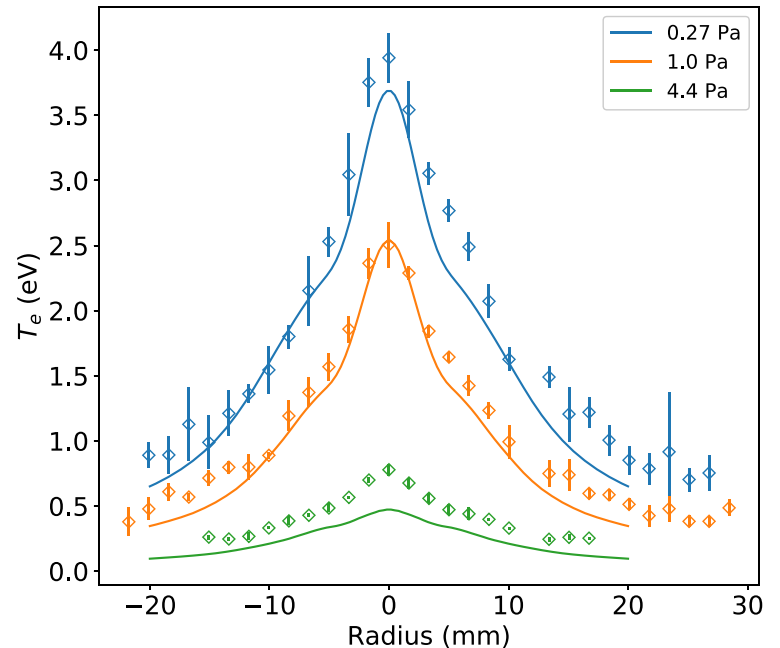

(b)

Figure 11. (a) Electron density and (b) temperature radial profiles from the high density case at $Z=0$ for $P_{\mathrm{n}}=0.45,1.0$ and $4.4 \mathrm{~Pa}$.

is shown for the peak electron density between the simulation and experimental measurements. In this regime there are no density rollovers, and both the simulation and experiment show the trend of increasing density. However, in the simulation, the density increases very slowly when gas pressure is increased. The radial profile comparison is shown in figure 11 for pressure values $0.27,1.0$ and $4.4 \mathrm{~Pa}$. In contrast to the previous case, here we observe the plasma to be widening with increased gas pressure. The simulated $T_{\mathrm{e}}$ profiles are in line with experimental data with the exception of the highest pressure point, while the simulated $n_{\mathrm{e}}$ profiles does not widen nor heighten for the mid pressure range. The increase in density is caused by the slowing down of plasma flow from the plasma source to target. The different behavior indicates that the particle flux in the simulation is underestimated, and the volumetric loss of plasma through recombination is able to compensate the particle accumulation that causes the density increase. Contrary to the high density case, here the plasma velocity at the source boundary might be underestimated.

\section{Electric current pathways and effects in Magnum-PSI detachment experiments}

Before we discuss the effects of increased plasma-neutral interaction as a mechanism for detachment, it is important to understand the behavior of electric currents in Magnum-PSI plasmas. Magnum-PSI utilizes a cascaded arc source to generate high flux low temperature plasmas. The source consists of a negatively biased cathode tip at the center of a channel surrounded by electrically insulated copper plates. Gas can be inserted from the inlet of the channel, which ends with a nozzle that serves as a grounded anode. When voltage is applied to the cathode, an electric current is established within the channel, ionizing the gas and producing plasma that flows through the nozzle. In the absence of a magnetic field, the current is short-circuited into the grounded anode. In the presence of a high magnetic field $(1.2 \mathrm{~T})$, the charged particles are radially confined and the electric current will travel along the plasma beam. The current will radially dissipate along the 


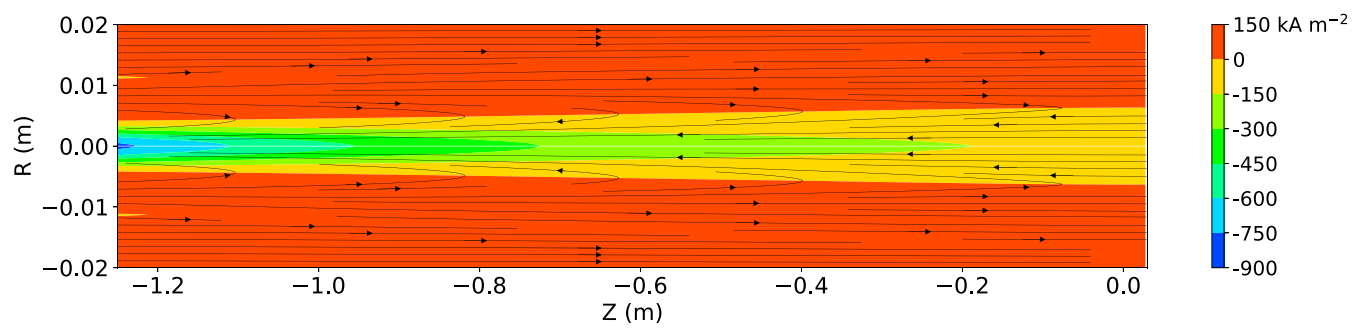

Figure 12. Streamlines of the current density in the plasma beam for the high density case at $P_{\mathrm{n}}=0.45 \mathrm{~Pa}$. Current is flowing from the grounded beam edge toward the target and returns toward the negatively biased center. The color indicates the magnitude of the current density.

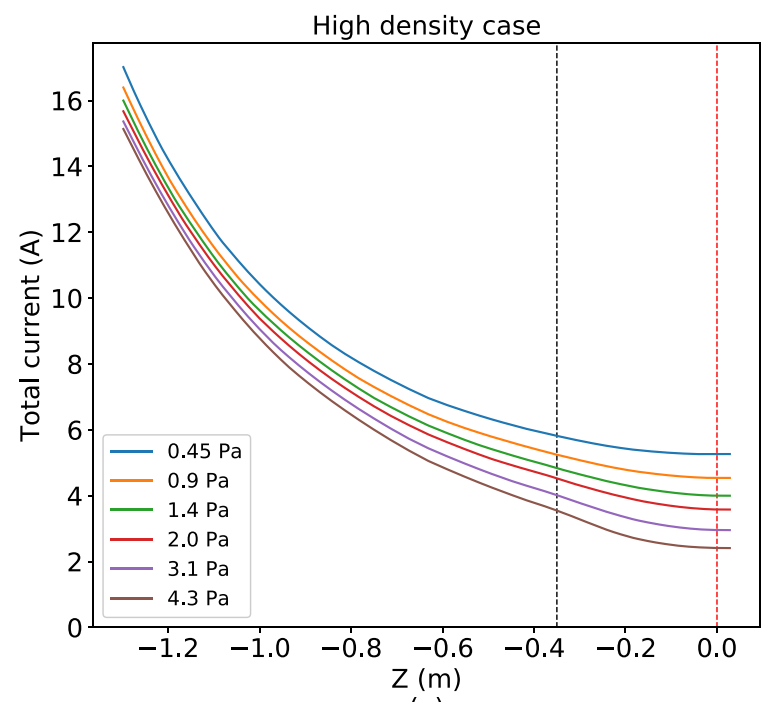

(a)

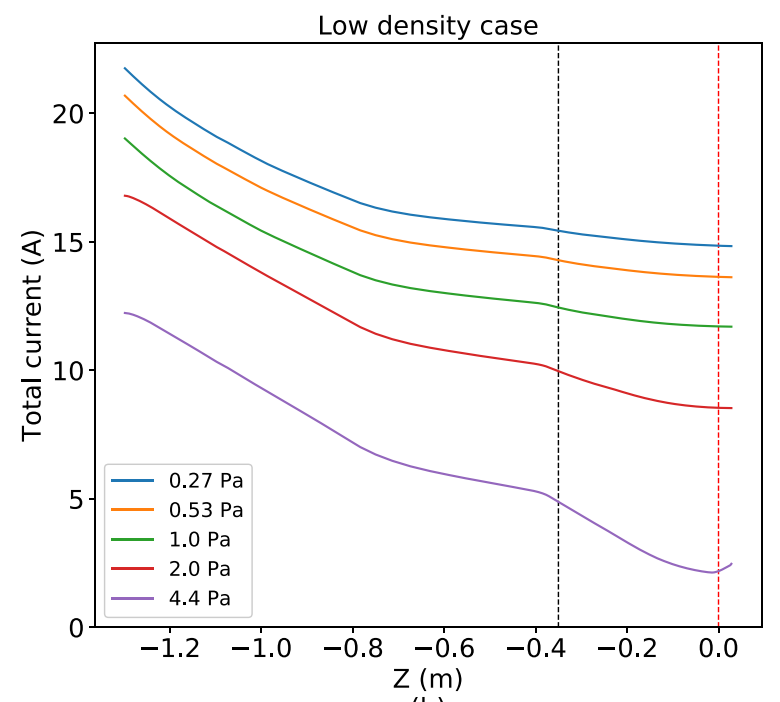

(b)

Figure 13. Total axial current in the plasma beam along the $Z$-axis. The red line indicates the location of the TS measurement during experiments. The black line indicates the location of the target chamber skimmer. (a) The high density case has peak negative bias of $-65 \mathrm{~V}$ at the source. (b) The low density case has peak negative bias of $-98 \mathrm{~V}$ at the source.

axial distance, however a substantial fraction can reach the target [26]. In our B2.5-Eunomia simulations, the electric current solution is governed by the potential boundary that is imposed at the source. Figure 12 shows the pathway of the current density in the plasma beam for the high density case at pressure $0.45 \mathrm{~Pa}$ in the target chamber. The current flows from the positively biased beam edge ( $r>5 \mathrm{~mm}$, see figure 5) at the source toward the floating target, and returns toward the negatively biased center. For all neutral pressure points $(0.27-4.4 \mathrm{~Pa})$, the pathways remain unchanged. A variation is observed instead in the magnitude of the current, as shown in figure 13. The low density case has higher current magnitude than the high density case as shown in figure 13 . The low density case has a higher electric field due to the more negatively bias potential boundary, thus resulting in a higher total current. This can also be the case in the experiment, considering that the electric current heats the plasma by Ohmic heating, and higher electron temperature was observed for the low density case. In the simulation, the contribution of Ohmic heating, compared to the plasma input energy, varies greatly between the high density case and the low density case. In the target chamber, the Ohmic heating amounts to about $4 \%$ of the plasma energy source for the high density case. In contrast, about $35 \%$ of the plasma energy source is consisted of Ohmic heating for the low density case. We observe a clear correlation between the increase in neutral background pressure and reduction in current. The amplitude of the change, however, differs greatly between the low density and high density case. For the high density case, the reduction is slight at the source, with values maintained around 15-17 A. The axial current decays exponentially toward the target, and there exists noticeable diminution within the target chamber, where the neutral pressure is varied. In contrast, the low density case shows heavy variation between points at different pressures along the beam. Here we also observe a slight enhancement of current decay within the target chamber, especially at high pressures. With the portion of Ohmic heating being relatively high in the low density case, the change in current can heavily impact the plasma energy balance. The reduction of axial current due to increasing gas pressure, as well as the difference of decay between the low density and high density case can be attributed to the decrease of current penetration from the plasma source. The length of penetration scales inversely with electron density and neutral density [26]. 

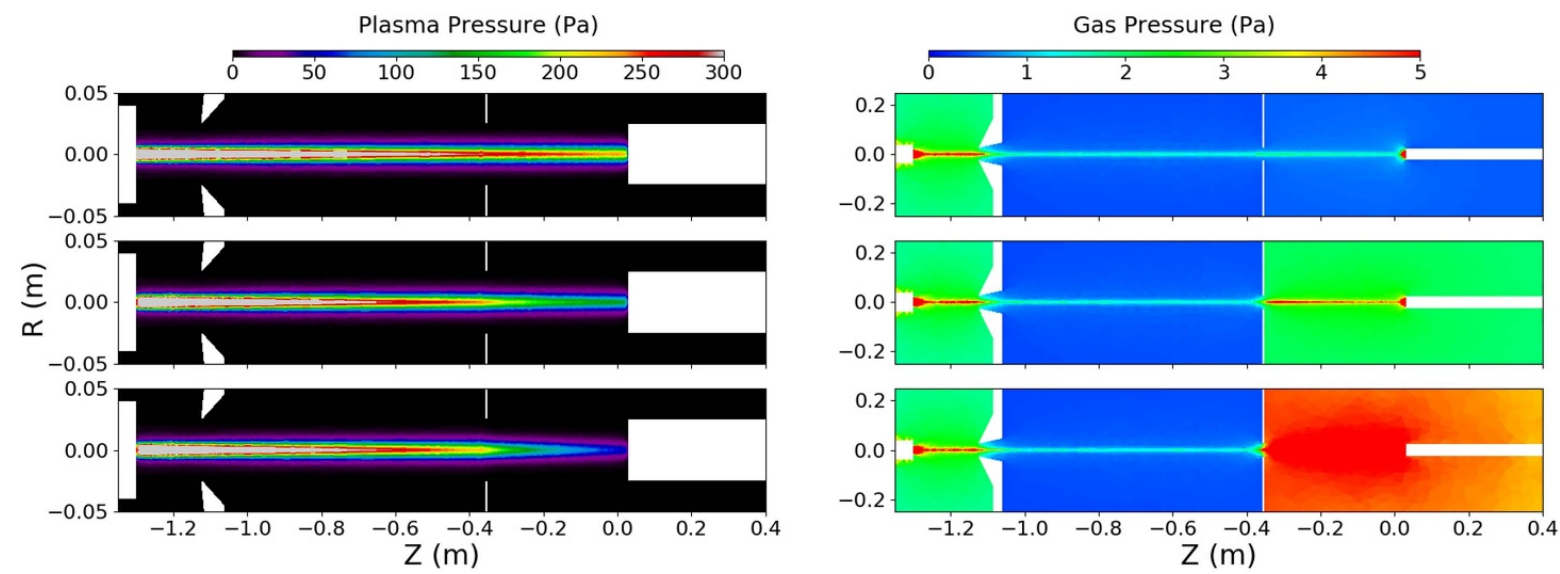

Figure 14. Two dimensional solution of the plasma pressure (left) and neutral pressure (right) from the high density case. From top to bottom the values of $P_{\mathrm{n}}=0.45,2.0$ and 4.3 Pa. The plasma pressure is reduced significantly within the target chamber where the neutral pressure is highest.

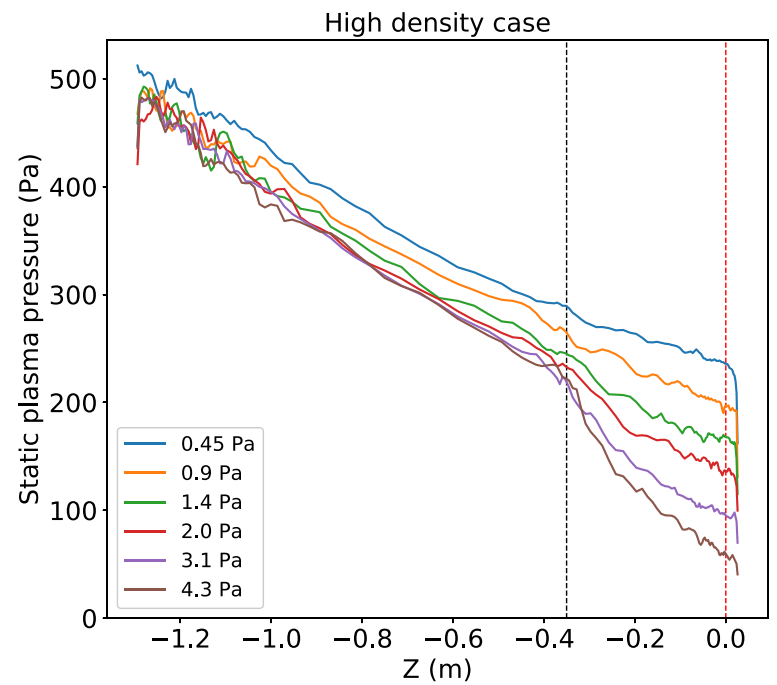

(a)

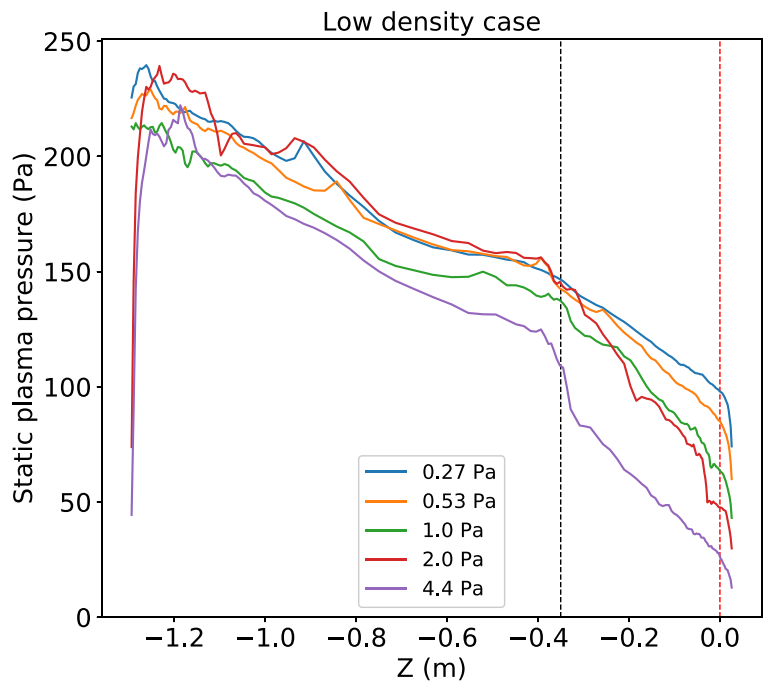

(b)

Figure 15. Static plasma pressure along the $Z$-axis of the plasma beam for (a) the high density case and (b) the low density case. The red vertical line indicates the location of the TS measurement during experiments. The black vertical line indicates the location of the target chamber skimmer. Both cases show plasma pressure gradient is increased with increasing $P_{\mathrm{n}}$ in the target chamber.

\section{Effects of neutral gas pressure in reducing static plasma pressure}

The results of B2.5-Eunomia simulations for Magnum-PSI detachment experiments and their comparison to experimental data have been discussed in section 5.2. Here we explore those results further to present a clearer picture on the effects of increasing plasma-neutral interaction on the characteristic behavior of detachment. One of those effects is the reduction of plasma pressure along the magnetic field direction. The total static plasma pressure, namely $p=n_{\mathrm{e}}\left(T_{\mathrm{e}}+T_{\mathrm{i}}\right)$, where $T_{\mathrm{i}}$ is the ion temperature, is shown for the high density case in figure 14. We observe significant plasma pressure loss within the target chamber. This is in conjunction with the increase of neutral gas pressure, $P_{\mathrm{n}}=n_{\mathrm{H}} T_{\mathrm{H}}+n_{\mathrm{H}_{2}} T_{\mathrm{H}_{2}}$, where $n_{\mathrm{H}}$ and $T_{\mathrm{H}}$ are the atomic hydrogen density and temperature respectively, and $n_{\mathrm{H}_{2}}$ and $T_{\mathrm{H}_{2}}$ are the molecular hydrogen density and temperature respectively. The neutral gas pressure is heavily localized in the target chamber as shown in figure 14. The localization of neutral pressure in the target chamber is maintained by the differential pumping, however the plasma flow effectively plugs the neutral flow at the skimmer hole, as shown in appendix B. Figure 15 shows the static plasma pressure along the center flux tube for all pressure cases to further elucidate the plasma pressure characteristics from source to target. For both the high and low density case, the plasma pressure decreases linearly for the zero puffing $(0.45$ and $0.27 \mathrm{~Pa}$ ) plasma condition from the plasma source to target. There is however, an enhancement of pressure loss within the target chamber as expected from the increasing neutral densities. With additional neutral background pressure, steeper gradients can be observed for the highest neutral pressures. The localization of this enhancement suggests that the plasma-neutral interaction is responsible for the plasma momentum loss. We also observe an offset decrease of plasma pressure for the entirety of the beam. This behavior corresponds to the different plasma current in each cases, 
which impacts the $T_{\mathrm{e}}$ and resulted in a slightly different $T_{\mathrm{e}}$ profile for each pressure cases. Some anomalies are found in the low density case. In the two highest neutral pressures, near the source, $n_{\mathrm{e}}$ is dramatically reduced, causing the dip of plasma pressure near that point. It is caused by unphysical velocity solutions (upward to Mach 3) near the source boundary. This numerical anomaly is caused by the over constraining of the source boundary. When the velocity boundary is reverted to the Neumann condition, the unphysical artifact disappears. As expected, this boundary type does not yield the same particle flux as before, with the difference of about $25 \%$ more particle flux. The fact that the same particle flux cannot be applied to the two highest pressure points in the low density case suggests that the source is being affected by the changes of neutral pressure in the target chamber. This effect is rather weak for the high density case as the simulation does not produce the same artifact near the source. While relaxing the velocity boundary solves the anomaly, relaxing other conditions instead seems to work as well. Ultimately, the effects can be identified by a future experiment where the plasma parameters near the source are measured while the target chamber pressure is increased. Nevertheless, the artifact is very localized within a few simulation cells near the source boundary, and the axial profile of the plasma pressure seems to recover downstream. Additionally, the plasma density and temperature profile excluding the problematic cells are similar in their axial shape and order of magnitude compared to the re-simulation using the Neumann boundary condition. For the purpose of investigating the effects of neutral gas in achieving detachment in the target chamber, we conclude that these solutions are preferred because the particle flux is equal.

In our simulations we have assumed that the collision rates (such as neutral-ion interactions) are reliable while adjusting the velocity profile, potential profile, and cross-field transport coefficients in order to fit the target-side density and temperature profile widths and heights. This is in contrast with a long-standing issue with SOLPS modeling of divertors, see for instance [27, 28], which both use neutral pressures approximately a factor 2 higher than the experimental pressure, in order to obtain solutions that are otherwise close to experimental. While that practice could be compensating for underestimated neutral-ion interactions, we do not know if this is the case, and we cannot be sure that this situation carries over to the linear plasma case of Magnum-PSI. Therefore, without claiming additional knowledge on this issue, we have to assume that the neutral pressure, which is one of the most reliably known quantities in Magnum-PSI, should be exactly matched by our simulations.

\section{Target particle and heat flux reduction in Magnum-PSI detachment experiments}

Other important aspects of detachment are reductions in particle and heat flux to the target. Here we describe the effects of increasing neutral gas pressure to the target particle and heat flux. In B2.5-Eunomia the particle and heat flux are calculated as follows:

$$
\begin{gathered}
\Gamma=n_{\mathrm{e}} v_{\mathrm{a}} \\
q_{\mathrm{e}}=\Gamma k T_{\mathrm{e}}-\kappa_{\mathrm{e}} \frac{\partial T_{\mathrm{e}}}{\partial z} \\
q_{\mathrm{i}}=\Gamma\left(k T_{\mathrm{i}}+e E_{\alpha}\right)-\kappa_{\mathrm{i}} \frac{\partial T_{\mathrm{i}}}{\partial z} \\
q=q_{\mathrm{e}}+q_{\mathrm{i}} .
\end{gathered}
$$

Here $\Gamma$ is the particle flux to the target, $n_{\mathrm{e}}$ is the electron density value in the cell in front of the target, $v_{\mathrm{a}}$ is the ion flow velocity at the target boundary, $q_{\mathrm{e}}$ and $q_{\mathrm{i}}$ are the electron and ion heat flux to the target respectively, $T_{\mathrm{e}}$ and $T_{\mathrm{i}}$ are the electron and ion temperature in front of the target respectively, and $\kappa_{\mathrm{e}}$ and $\kappa_{\mathrm{i}}$ are the electron and ion thermal diffusivity, respectively. To compare the target fluxes in the simulation with experiments mentioned in section 2, the particle and heat flux to the target are extrapolated using the TS data at $Z=0$ with the following formula [29], assuming $T_{\mathrm{e}}=T_{\mathrm{i}}$ and no neutral friction:

$$
\begin{aligned}
\Gamma_{\mathrm{ext}} & =\frac{1}{2} n_{\mathrm{e}, z=0} \sqrt{\frac{(1+\gamma) k T_{\mathrm{e}, z=0}}{m_{p}}} \\
q_{\mathrm{ext}} & =\Gamma_{\mathrm{ext}}\left(\gamma_{s h} k T_{\mathrm{e}, z=0}+e E_{\alpha}\right) .
\end{aligned}
$$

Here $\Gamma_{\text {ext }}$ is the extrapolated particle flux to the target, $n_{\mathrm{e}, z=0}$ and $T_{\mathrm{e}, z=0}$ are the electron density and temperature measured with TS at $Z=0, \gamma=\frac{5}{3}$ assuming adiabatic flow, $q_{\text {ext }}$ is the extrapolated heat flux to the target, $m_{p}$ is the proton mass, $\gamma_{s h}=7$ is the sheath heat transmission coefficient, and $E_{\alpha}$ (in $\mathrm{eV})$ is the hydrogen ionization potential $(13.6 \mathrm{eV})$. The integrated particle and heat flux from simulations and extrapolation of TS data are shown in figures 16 and 17. For the high density case, the particle and heat flux to the target are reduced by $62 \%$ and $66 \%$ respectively from the maximum observed values by increasing the gas pressure to $4.3 \mathrm{~Pa}$. This is close to the approximation with TS data of about $72 \%$ for the particle flux and $77 \%$ for the heat flux. While the values themselves are in good agreement, caution is warranted since there is discrepancy between the measured electron density, $n_{\mathrm{e}, z=0}$ used in (5), and the simulation results shown in figure 8 . When using simulation values for $n_{\mathrm{e}, z=0}$ and $T_{\mathrm{e}, z=0}$ in (5), $\Gamma_{\mathrm{ext}}$ is larger by a factor of two compared to $\Gamma$. The larger particle flux corresponds to the discrepancy in $n_{\mathrm{e}, z=0}$ profile. This is also reflected by the heat flux shown in figure 17(a), where a significant part is contributed by the plasma particle flux times the ionization potential. In contrast with the high density case, the low density case resulted in a lower simulated particle and heat flux at the target compared to the extrapolation using TS data. Using (5) with low density case values for $n_{\mathrm{e}, z=0}$ and $T_{\mathrm{e}, z=0}, \Gamma_{\text {ext }}$ is a factor of two lower than $\Gamma$, and consequently the heat flux $q_{\text {ext }}$ is lower than $q$. Again, the discrepancy is related to the $n_{\mathrm{e}, z=0}$ profile, especially at $5<R<15 \mathrm{~mm}$, where the contribution to the particle flux is largest. For both cases, it shows that the simulations does not reflect the particle content at the 


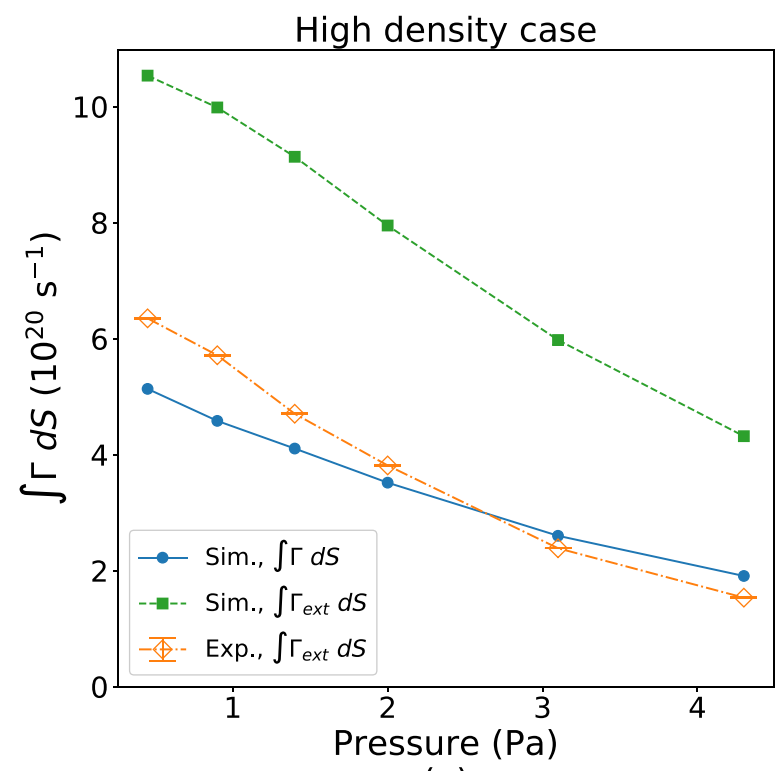

(a)

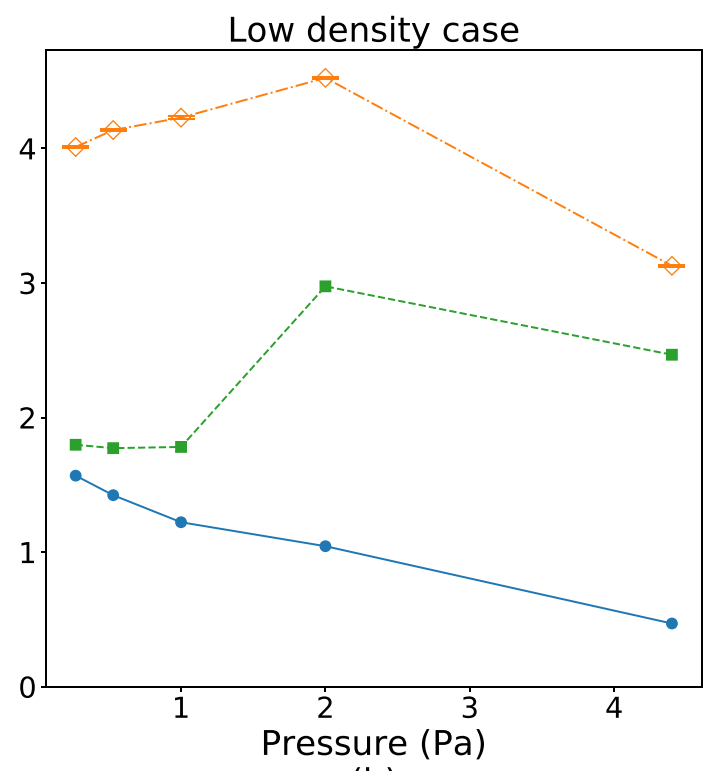

(b)

Figure 16. The plasma particle flux to the target integrated over the plasma beam width $S$ for the (a) high density case and (b) low density case. The simulated target particle flux, $\Gamma$, is derived from plasma parameters in front of the target using (1), and $\Gamma_{\text {ext }}$ is the target particle flux extrapolated from plasma parameters at $Z=0$ using (5). Here two $\Gamma_{\text {ext }}$ is shown, one using plasma parameters from TS (Exp.) and the other using plasma parameters from the simulation (Sim.).

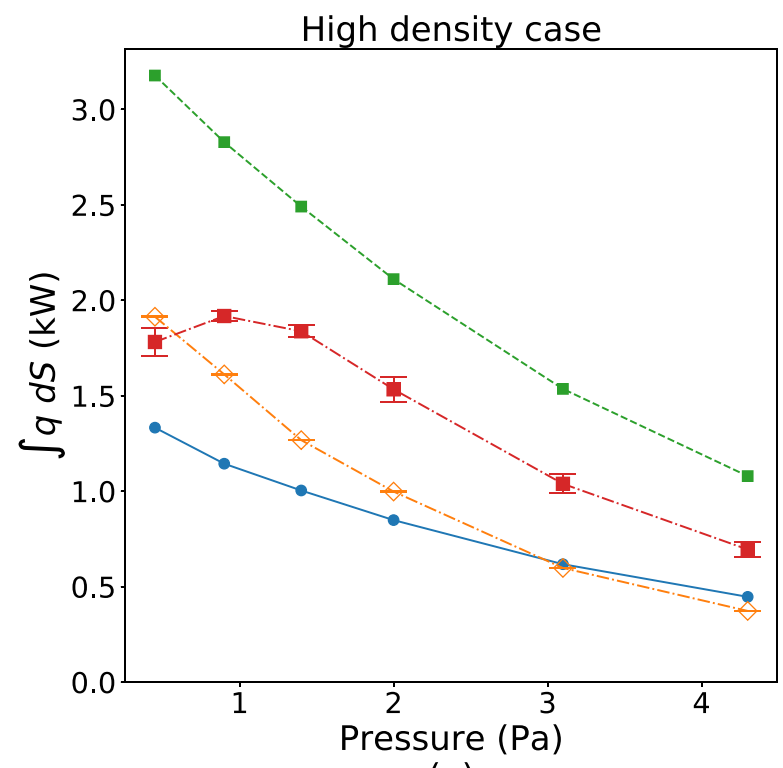

(a)

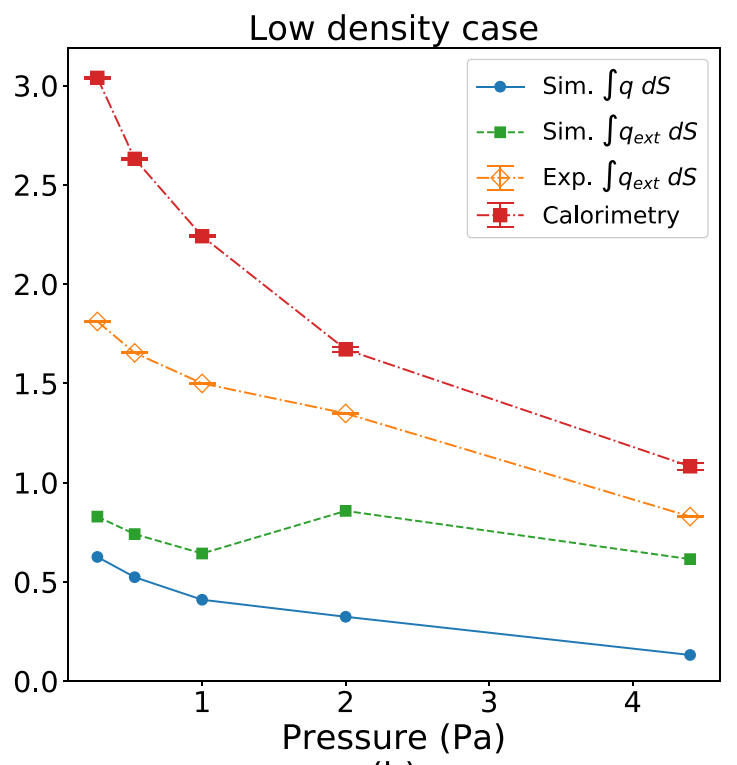

(b)

Figure 17. The plasma heat flux to the target integrated over the plasma beam width $S$ for the (a) high density case and (b) low density case. The simulated target heat flux, $q$, is derived from plasma parameters in front of the target using (4), and $q_{\text {ext }}$ is the target particle flux extrapolated from plasma parameters at $Z=0$ using (6). Here two $q_{\mathrm{ext}}$ is shown, one using plasma parameters from TS (Exp.) and the other using plasma parameters from the simulation (Sim.). The power reaching the target is also measured using calorimetry.

target TS location in the experiment. However, the difference between the two Sim. profiles shown in figures 16 and 17 suggest that the extrapolation using (5) and (6) may be overestimating the true flux reaching the target. One possibility is that neutral friction cannot be assumed to be non-existing between $Z=0$ and the target. In figure 17 , the calorimetry data from each cases is shown. Discrepancies between the calorimetry data and experimental $q_{\text {ext }}$ are observed, with calorimetry data measuring higher heat flux for both cases. The difference between calorimetry data and simulation results ( $q$ and $q_{\text {ext }}$ ) can be explained by the different particle content as discussed previously with the particle flux. It is suspected that additional heating may occur at the target by the current loop that is flowing from the source as described in figure 12. The heating fraction by this current is hard to isolate from the calorimetry data, since experimentally it is difficult to know the actual heat 
flux from the plasma that reaches the target. From the simulation, the heating power from this current can be calculated by using the current value at the target shown in figure 13 and the maximum radial potential difference at the target. It is calculated that for the low density case, the contribution from target current heating is roughly about $20 \%$ of the plasma heat flux from (6), and decreasing with increasing $P_{\mathrm{n}}$ as the total current decreases. This could explain the larger discrepancy in low $P_{\mathrm{n}}$ and the reduction in high $P_{\mathrm{n}}$. For the high density case, the radial potential difference is minimal, and the current heating contribution is negligible. For the low density case, the simulation overestimates the relative particle and heat reduction, at $70 \%$ (41\% for TS data) and 79\% (55\% for TS data) respectively. While the simulation fails to quantitatively capture the particle and heat flux to the target measured in the experiment, the effects of increasing neutral pressure to reduce the heat and particle flux are still replicated qualitatively.

\section{Conclusion and outlook}

This paper is the first part of studying the detachment experiment in Magnum-PSI. These experiments have been carried out to investigate the role of high gas pressure in reducing the intense heat and particle bombardment of plasma facing materials. The plasma parameters measured in said experiments have been successfully recreated in low and high plasma density regime, using B2.5-Eunomia simulations. Reasonable agreement is quantitatively achieved between the electron temperature measurement using TS and the simulation results, with some discrepancies in the electron density. Additional diagnostics can further provide input for B2.5-Eunomia in the future and thus can limit the necessary adjustment or assumptions, and subsequently improved the discrepancies found in this paper. One example is the usage of Collective TS [30] to measure the plasma flow velocity near the source, hence eliminating the need to use homogeneous Neumann boundary condition for the momentum equation. Another important example is the ability to simultaneously measure the electron density and temperature near the source and target, thus the effect of significant gas pressure addition to the source plasma parameters can be directly observed. There is also the possibility of plasma particle sinks that are not yet included, such as radial transport as a function of neutral density, or the inclusion of new collision pathways that remove plasma particles. The former can be implemented in a future revision of B2.5Eunomia, while the latter will be investigated in the second part of this study.

B2.5-Eunomia simulations presented here have given insight into the effects of increased gas pressure in the target chamber to the electric current characteristics of the plasma. The increase of gas pressure in the target chamber reduces the overall current penetration from the source and therefore can reduce the total current available for Ohmic heating. In essence, the loss of magnitude in current has an impact in energy loss for the detachment scenarios in Magnum-PSI, especially at high temperature regimes, and care should be taken when determining the contribution of plasma-neutral interaction in causing energy loss.

The key characteristics of Magnum-PSI detachment experiments, namely the loss of plasma pressure along the magnetic field and the reduction of particle and heat flux to the target, have been qualitatively reproduced with B2.5-Eunomia. The increase of gas pressure in the target chamber causes an increase of the plasma pressure gradient. This enhancement is localized near the target where the increase of plasma-neutral interaction is expected due to the increase in neutral density. We also observed the reduction of particle and heat flux to the target as a function of increased plasma-neutral interaction. While both cases have demonstrated significant plasma flux reduction, different collision processes can occur due to the different plasma parameters between the cases. As the second part of studying Magnum-PSI detachment experiment we will perform a detailed analysis of the exact contribution of individual plasma-neutral collisions responsible for particle and heat flux reduction to the target. This study will be reported in a companion paper.

\section{Data availability statement}

The data that support the findings of this study are openly available in Zenodo at https://dx.doi.org/10.5281/ zenodo.4462972, reference number [31].

\section{Acknowledgments}

Differ is part of the institutes organization of NWO. This work is part of the research programme Taming the Flame, which is partly financed by NWO. This work has been carried out within the framework of the EUROfusion Consortium and has received funding from the Euratom research and training programme 2014-2018 and 2019-2020 under Grant Agreement No. 633053. The views and opinions expressed herein do not necessarily reflect those of the European Commission. This work was carried out on the Dutch national e-infrastructure with the support of SURF Cooperative. We acknowledge computing resources provided on the MarconiFusion HPC infrastructure.

\section{Appendix A. Eunomia: collision formulas}

The list of neutral and charged particle collisions included in the Eunomia code used for simulations described in this paper can be found in table A1. 
Table A1. Collisions used in the Eunomia code. The databases used are HYDHEL [32], AMJUEL [33], and H2VIBR [34]. For

collisions between neutral particles the cross-section are calculated using the Lennard-Jones potential of the atom or molecule [35]. Spontaneous means the process occurs simultaneously when the ionic reactant $\left(\mathrm{H}_{2}^{+}\right.$and $\left.\mathrm{H}^{-}\right)$is produced by another process. Collisions involving vibrational states of $\mathrm{H}_{2}$ occur for each of the corresponding states that are simulated as test particles in Eunomia.

\begin{tabular}{ll}
\hline Collision formula & Database \\
\hline $\mathrm{H}+e^{-} \rightarrow \mathrm{H}^{+}+2 e^{-}$ & HYDHEL \\
$\mathrm{H}+e^{-} \rightarrow \mathrm{H}^{*}(n=2)+e^{-}$ & HYDHEL \\
$\mathrm{H}+\mathrm{H}^{+} \rightarrow \mathrm{H}^{+}+\mathrm{H}$ & HYDHEL \\
$\mathrm{H}+\mathrm{H} \rightarrow$ elastic & Lennard-Jones \\
$\mathrm{H}+\mathrm{H}_{2} \rightarrow$ elastic & Lennard-Jones. \\
$\mathrm{H}_{2}+\mathrm{H}_{2} \rightarrow$ elastic & Lennard-Jones. \\
$\mathrm{H}+\mathrm{H}^{+} \rightarrow$ elastic & AMJUEL \\
$\mathrm{H}_{2}+\mathrm{H}^{+} \rightarrow$ elastic & AMJUEL \\
$\mathrm{H}^{+}+e^{-} \rightarrow \mathrm{H}$ & AMJUEL \\
$\mathrm{H}^{+}+\mathrm{H}_{2}(v=0-14) \rightarrow \mathrm{H}+\mathrm{H}_{2}^{+}$ & H2VIBR \\
$\mathrm{H}_{2}^{+}+e^{-} \rightarrow \mathrm{H}+\mathrm{H}^{*}$ & Spontaneous \\
$\mathrm{H}_{2}(v=i)+e^{-} \rightarrow \mathrm{H}_{2}(v=i+1)+e^{-}$ & H2VIBR \\
$\mathrm{H}_{2}(v=i)+e^{-} \rightarrow \mathrm{H}_{2}(v=i-1)+e^{-}$ & H2VIBR \\
$\mathrm{H}_{2}(v=0-14)+e^{-} \rightarrow \mathrm{H}+\mathrm{H}^{-}$ & H2VIBR \\
$\mathrm{H}^{-}+\mathrm{H}^{+} \rightarrow \mathrm{H}+\mathrm{H}^{*}$ & Spontaneous \\
$\mathrm{H}_{2}(v=0-14)+e^{-} \rightarrow \mathrm{H}+\mathrm{H}^{+}+e^{-}$ & H2VIBR \\
\hline
\end{tabular}

\section{Appendix B. Plasma plugging effect}

As mentioned in section 3, the model utilizes a feedback boundary condition for handling the neutral pressure in each chambers. The pressure at the source and the beam dump chamber are kept stationary while the pressure at the target chamber is increased. Together with the skimmers in place, the pressure between the chambers are effectively localized as shown in figure 14. This is especially true for the highest pressure points both for experiment A and B. At first intuition, there should be a high flux of neutral particles leaking from the target chamber to the beam dump chamber due to the pressure gradient. However, due to the presence of the plasma, the skimmer opening is 'plugged', thereby reducing the effective neutral flux. To test this, we run the simulation without any plasma for the experiment B case at $4.3 \mathrm{~Pa}$. Figure B1 shows the radial profiles of particle flux density at the target skimmer opening. With the plasma, most of atomic hydrogen flows into the target chamber. This is expected since the bulk of atomic hydrogen flux is coming from the plasma source and the flow velocity is equal to the ion flow velocity. In contrast, without any plasma, the net particle flux is zero. This is also expected considering there is no $\mathrm{H}$ source due to plasma surface recombination at the target, and there is no net flow velocity at the source. What is interesting, however, is the contrast between particle fluxes of $\mathrm{H}_{2}$. Since $\mathrm{H}_{2}$ has a dedicated puffing source to produce the required $4.3 \mathrm{~Pa}$ pressure, without any impedance there should be heavy flow of $\mathrm{H}_{2}$ from the target chamber to the heating chamber, and this is shown in red in figure B1. With the plasma, the molecular flow experiences drag and is significantly reduced, hence resulting in a plugging effect.

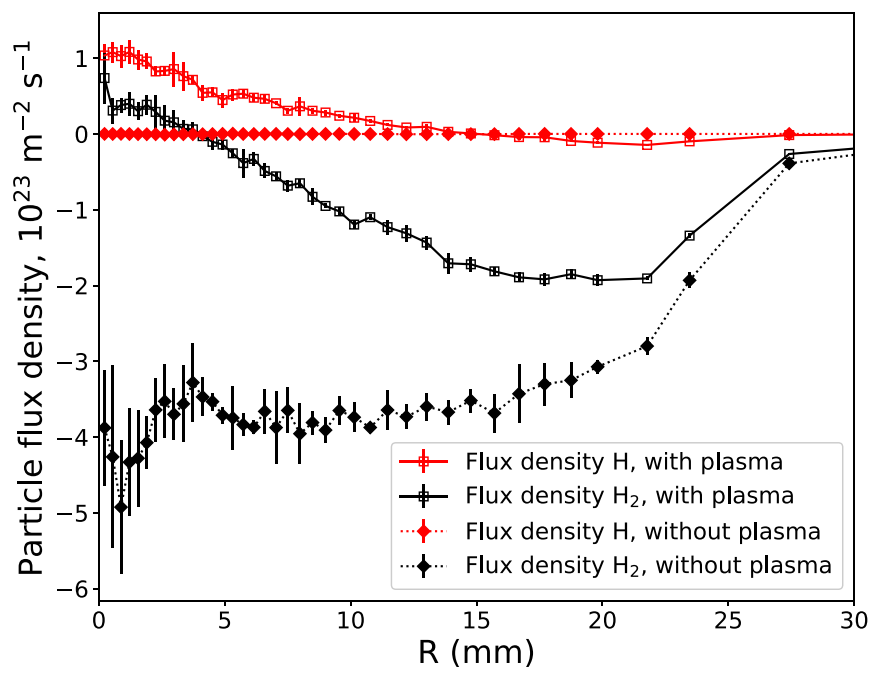

Figure B1. Radial profiles of neutral particle flux at the target skimmer opening for experiment $\mathrm{B}$ case 4.3 Pa. Positive values correspond to flow direction into the target chamber.

\section{ORCID iDs}

R Chandra (D) https://orcid.org/0000-0001-6098-3756

P Diomede (1) https://orcid.org/0000-0002-4523-3049

H J N van Eck (D) https://orcid.org/0000-0002-1166-8837

H J van der Meiden (iD https://orcid.org/0000-0002-55854279

T W Morgan (D) https://orcid.org/0000-0002-5066-015X

J W M Vernimmen (D) https://orcid.org/0000-0003-26987459

E Westerhof (D) https://orcid.org/0000-0002-0749-9399

\section{References}

[1] Loarte A et al 1998 Nucl. Fusion 38 331-71

[2] Krasheninnikov S I 1998 Czech. J. Phys. 48 97-112

[3] Stangeby P 2020 Plasma Phys. Control. Fusion 62025012

[4] Kotov V and Reiter D 2009 Plasma Phys. Control. Fusion 51115002

[5] Reimold F et al 2015 J. Nucl. Mater. 463 128-34

[6] Wensing M et al 2019 Plasma Phys. Control. Fusion 61085029

[7] Sang C, Guo H, Stangeby P, Lao L and Taylor T 2017 Nucl. Fusion 57056043

[8] Moulton D, Harrison J, Lipschultz B and Coster D 2017 Plasma Phys. Control. Fusion 59065011

[9] Park J S, Groth M, Pitts R, Bak J G, Thatipamula S, Juhn J W, Hong S H and Choe W 2018 Nucl. Fusion 58126033

[10] Ohno N 2017 Plasma Phys. Control. Fusion 59034007

[11] Hsu W L, Yamada M and Barrett P J 1982 Phys. Rev. Lett. 49 1001-4

[12] van Eck H et al 2019 Fusion Eng. Des. 142 26-32

[13] Pitts R A, Kukushkin A, Loarte A, Martin A, Merola M, Kessel C E, Komarov V and Shimada M 2009 Phys. Scripta T138 014001

[14] Perillo R, Akkermans G, Classen I, Vijvers W, Chandra R, Jesko K, Korving S, Vernimmen J and de Baar M 2019 Nucl. Mater. Energy 19 87-93 
[15] Wieggers R C, Coster D P, Groen P W C, de Blank H J and Goedheer W J 2013 J. Nucl. Mater. 438 S643-6

[16] Schneider R, Bonnin X, Borrass K, Coster D P, Kastelewicz H, Reiter D, Rozhansky V A and Braams B J 2006 Contrib. Plasma Phys. 46 3-191

[17] Jesko K 2018 Studying Divertor Relevant Plasmas in Linear Devices PhD dissertation Technische Universiteit Eindhoven

[18] Perillo R, Chandra R, Akkermans G R A, Classen I G J and Korving S Q 2019 Phys. Plasmas 26102502

[19] Bonnin X, Dekeyser W, Pitts R, Coster D, Voskoboynikov S and Wiesen S 2016 Plasma Fusion Res. 111403102

[20] Wieggers R C 2012 B2.5-Eunomia simulations of Pilot-PSI $\mathrm{PhD}$ Dissertation Technische Universiteit Eindhoven

[21] Schneider R, Coster D, Braams B, Xantopoulos P, Rozhansky V, Voskoboynikov S, Kovaltsova L and Bürbaumer H 2000 Contrib. Plasma Phys. $40328-33$

[22] Vijvers W A, Schram D C, Shumack A E, Lopes Cardozo N J, Rapp J and Van Rooij G J 2010 Plasma Sources Sci. Technol. 19065016

[23] Shumack A E et al 2008 Phys. Rev. E 78046405
[24] Ješko K, Marandet Y, Bufferand H, Gunn J P, van der Meiden H J and Ciraolo G 2018 Plasma Phys. Control. Fusion 60125009

[25] Braginskii S I 1965 Transport processes in plasma M A Leontovich Rev. Plasma Phys. (Consultants Bureau: New York, NY) 1 205-311

[26] Shumack A E, de Blank H J, Westerhout J and van Rooij G J 2012 Plasma Phys. Control. Fusion 54125006

[27] Kotov V, Reiter D, Pitts R A, Jachmich S, Huber A and Coster D P 2008 Plasma Phys. Control. Fusion 50105012

[28] Shafer M et al 2019 Nucl. Mater. Energy 19 487-92

[29] Stangeby P C 2000 The plasma boundary of magnetic fusion devices Institute of Physics Pub. Philadelphia, Pennsylvania

[30] van der Meiden H J 2010 Plasma Phys. Control. Fusion 52045009

[31] Chandra R 2021 (https://doi.org/10.5281/zenodo.4462972)

[32] Reiter D 2013 The data file HYDHEL (Version March 2013)

[33] Reiter D 2011 The data file AMJUEL (Version December 12, 2011)

[34] Reiter D 2005 The data file H2VIBR (Version April 2005)

[35] Perrin J, Leroy O and Bordage M 1996 Contrib. Plasma Phys. $363-49$ 